\title{
Differential Histopathological and Behavioral Outcomes Eight Weeks after Rat Spinal Cord Injury by Contusion, Dislocation, and Distraction Mechanisms
}

\author{
Kinon Chen,1,4,6 Jie Liu,,* Peggy Assinck,1,2,* Tim Bhatnagar,1,3 Femke Streijger, Qingan Zhu,1,4 \\ Marcel F. Dvorak, ${ }^{1,4}$ Brian K. Kwon, ${ }^{1,4}$ Wolfram Tetzlaff, ${ }^{1,5, * *}$ and Thomas R. Oxland ${ }^{1,3,4, * *}$
}

\begin{abstract}
The objective of this study was to compare the long-term histological and behavioral outcomes after spinal cord injury (SCI) induced by one of three distinct biomechanical mechanisms: dislocation, contusion, and distraction. Thirty male SpragueDawley rats were randomized to incur a traumatic cervical SCI by one of these three clinically relevant mechanisms. The injured cervical spines were surgically stabilized, and motor function was assessed for the following 8 weeks. The spinal cords were then harvested for histologic analysis. Quantification of white matter sparing using Luxol fast blue staining revealed that dislocation injury caused the greatest overall loss of white matter, both laterally and along the rostrocaudal axis of the injured cord. Distraction caused enlarged extracellular spaces and structural alteration in the white matter but spared the most myelinated axons overall. Contusion caused the most severe loss of myelinated axons in the dorsal white matter. Immunohistochemistry for the neuronal marker NeuN combined with Fluoro Nissl revealed that the dislocation mechanism resulted in the greatest neuronal cell losses in both the ventral and dorsal horns. After the distraction injury mechanism, animals displayed no recovery of grip strength over time, in contrast to the animals subjected to contusion or dislocation injuries. After the dislocation injury mechanism, animals displayed no improvement in the grooming test, in contrast to the animals subjected to contusion or distraction injuries. These data indicate that different SCI mechanisms result in distinct patterns of histopathology and behavioral recovery. Understanding this heterogeneity may be important for the future development of therapeutic interventions that target specific neuropathology after SCI.
\end{abstract}

Key words: animal models; behavior; biomechanics; histology; injury mechanisms; spinal cord injury

\section{Introduction}

Q PINAl CORD INJURY (SCI) is devastating to the injured persons $\checkmark$ and very costly to our society. ${ }^{1-3}$ Despite decades of basic science and clinical research, treatments that convincingly promote improved spinal cord function after traumatic injury in humans remain elusive. ${ }^{4}$ In his seminal review article on clinical trials for SCI, Tator" noted that the "disregard for the heterogeneity of human SCI may be an important reason for the failure to find clinically effective therapies."

One aspect of this heterogeneity relates to the morphological pattern of the injury to the spinal column that results in a particular SCI mechanism. The two most common spinal column injury pat- terns are fracture-dislocations (45\%) and burst fracture (40\%). ${ }^{1,2}$ These patterns of spinal column injury result in different mechanical insults to the spinal cord, with a fracture dislocation subjecting the spinal cord to a shear or dislocation injury mechanism, a burst fracture producing a contusion injury mechanism to the spinal cord, and hyperextension or hyperflexion injuries creating some degree of distraction or longitudinal tension on the spinal cord. ${ }^{6}$ The flexiondistraction and hyperextension injuries that occur in both adult and pediatric spinal injuries may subject the spinal cord to a pure distraction force without contusion or shear. ${ }^{7-10}$

Analyses of how these distinct morphological patterns of spinal column injury influence the injury to the spinal cord have demonstrated that the internal spinal cord deformations are grossly

\footnotetext{
${ }^{1}$ International Collaboration on Repair Discoveries (ICORD), ${ }^{2}$ Graduate Program in Neuroscience, Departments of ${ }^{3}$ Mechanical Engineering, ${ }^{4}$ Orthopaedics, ${ }^{5}$ Zoology and Surgery, University of British Columbia, Vancouver, British Columbia, Canada.

${ }^{6}$ School of Biological Science and Medical Engineering, Beihang University, Haidian, Beijing, China.

*Equal contribution second authors.

**Co-senior authors.

(c) Kinon Chen, et al., 2016; Published by Mary Ann Liebert, Inc. This Open Access article is distributed under the terms of the Creative Commons Attribution Noncommercial License (http://creativecommons.org/licenses/by-nc/4.0/) which permits any noncommercial use, distribution, and reproduction in any medium, provided the original author(s) and the source are credited.
} 
different. Thus one would anticipate that the damage to the cord would also vary with the different SCI mechanisms. ${ }^{11,12}$

A key question is whether these different patterns of spinal column injury that are commonly observed in human patients with SCI result in distinct histopathologic features within the injured spinal cord and ultimately different functional outcomes. Such heterogeneity in the histopathology of the injured spinal cord could contribute to the difficulties in demonstrating functional benefit with therapies that have a specific and narrow therapeutic target. In addition, such differences may need to be considered in the scientific development of SCI treatments and the clinical decisions on which types of injuries are most likely to benefit from specific treatments. 5,13

The experimental severity and extent of SCI that occurs with these various morphological patterns of spinal column injury have not been critically analyzed. Contusion remains the gold standard experimental mechanism of SCI and was pioneered over 100 years ago in the studies by Allen. ${ }^{14}$ In an experimental contusion injury, the pathophysiology within the rodent spinal cord is well characterized-consisting of a central cavitation, surrounded by glial scar and a region of disrupted, demyelinated, and intact axons around the ventral and lateral periphery.

The dislocation mechanism of SCI, despite being the most common clinically, was studied first in animals by Fiford and associates ${ }^{15}$ for a lateral dislocation and by Choo and colleagues ${ }^{6}$ for an anteriorposterior dislocation. Both of these studies suggested that the spinal cord after a dislocation included the characteristic central cavitation seen in the contusion injury, but also included extensive damage to the axons in the spared rim. The distraction mechanism of SCI produces a more diffuse pattern of damage, as described previously. ${ }^{6,16-18}$

Our group is the only one to compare directly the contusion, dislocation, and distraction SCI mechanisms and in those studies, many distinct histological differences were observed at $5 \mathrm{~min}$ and $3 \mathrm{~h}$ post-injury. ${ }^{6,19}$ Therefore, the aims of the present study were to examine the histopathological and behavioral outcomes across contusion, dislocation, and distraction SCI mechanisms after mediumterm survival (i.e., 8 weeks) in a rat model.

\section{Methods}

\section{Animals}

All procedures were approved by the University of British Columbia (UBC) Committee on Animal Care in accordance with the Guide to the Care and Use of Experimental Animals by the Canadian Council on Animal Care. ${ }^{20}$ Thirty 16-day-old male Sprague-Dawley (SD) rats were purchased within the university. Male rats were chosen because of the male preponderance for SCI clinically. ${ }^{1}$ The animals were acclimated to our facility at $22^{\circ} \mathrm{C}$ and indoor humidity $(30-50 \%)$ on a reverse light cycle $(12 \mathrm{~h} / 12 \mathrm{~h})$ with standard chow and filtered water ad libitum and handled in the first 5 days. An additional five $340 \mathrm{~g}$ male SD rats were used as uninjured controls for the histological analysis (i.e., weight-matched to injured rats).

\section{Behavior}

The animals were trained to perform several behavioral tasks for the next 17 days. The Martinez locomotor rating scale was used to assess the forelimb and hindlimb locomotor functions after cervical SCI based on movements of forelimb and hindlimb articulations, weight support of the limbs, digit position, stepping, forelimbhindlimb coordination, and tail position in an open-field arena. ${ }^{21}$ The forelimb locomotor assessment scale (FLAS) was used to assess forelimb dysfunction based on predominance of the joints, digit position, paw placement, forelimb activity, four-limb coordination, and balance during alley crossing. ${ }^{22}$
The grooming test was used to assess forelimb grooming function based on the ability of the animals to contact the paw with any part of the face or head after water was applied to their head and back. $^{23}$ The grip strength test measured the maximum grip strength when the animals grasped a metal bar with each forelimb and was steadily pulled away until the grip was broken. ${ }^{24}$ The Montoya staircase was used to assess skilled forelimb reaching and grasping by measuring the number of pellets taken and pellets eaten over a $15 \mathrm{~min}$ period with the animal contained within a staircase box with left and right stairs filled with color-coded food pellets. ${ }^{25}$

The ladder rung walking test was used to assess skilled walking by measuring forelimb and hindlimb placing, stepping, and interlimb coordination, when the animals walked along a horizontal ladder with unevenly spaced rungs. ${ }^{26}$ The CatWalk Gait Analysis (Noldus Information Technology, Wageningen, The Netherlands) was used to perform an automated quantitative gait analysis during walkway crossing. ${ }^{27}$

Each task was performed at the same time of day during the dark cycle with at least $4 \mathrm{~h}$ of rest between the tasks. The Martinez locomotor rating scale and the FLAS were performed on the same day. In the Martinez locomotor rating scale, the scoring spreadsheet, originally for unilateral injury, was expanded to evaluate bilateral injury. In the FLAS, nonfunctional, partially functional, and normal digit positions would earn a score of 0,1 , and 2 (i.e., instead of 1, 2, and 3) respectively, and nonplantar paw placement would earn no score (i.e., instead of 1), so that the scaling system was adjusted from 4-64 to 0-60. The grip strength and the ladder rung walking test were always the last task of the day. The Montoya staircase was always the only task of the day, and in preparation for this test, the animals were fasted for $14 \mathrm{~h}$ before the task to motivate them. The food pellets in the top two stairs were neglected in the analysis, because the animals could reach these pellets with the tongue. ${ }^{28}$

The animals accomplished five trials of the Montoya staircase and four trials of each of the other tasks at the end of the training period. Baseline assessment was performed in the following 4 days. Puffed wheat and nutrition shake were provided to the animals in the next 3 days of rest before surgery and also after surgery.

\section{Surgery/injury production}

The animals were 46 days old and $282 \pm 17 \mathrm{~g}$ at the time of surgery. They were anesthetized with inhalational isoflurane. Their backs were shaved and disinfected. Analgesics pre-medication of $2 \%$ lidocaine (subcutaneous [SC]) $0.5 \mathrm{~mL}$ into the area of skin incision) and buprenorphine $(0.05 \mathrm{mg} / \mathrm{kg}$ in $0.5 \mathrm{~mL}, \mathrm{SC})$ were given to the animals and Ringer lactate $(10 \mathrm{~mL}, \mathrm{SC})$ was also injected to prevent dehydration. Lacrilube ophthalmic ointment was applied to their eyes to prevent the eyes from drying.

The animals were kept on a heating pad between $36-37^{\circ} \mathrm{C}$ to avoid hypothermia during surgery. Animal temperature, heart rate, and blood oxygenation were monitored throughout surgery.

A cervical SCI was induced because of the preponderance of cervical injuries in clinical SCI. ${ }^{1,2}$ The animals' spinal columns from $\mathrm{C} 2$ to $\mathrm{C} 7$ were exposed dorsally by incision and splitting the muscles along the midline. Moderate contusion $(n=8)$, dislocation $(n=11)$, and distraction $(n=11)$ injuries were produced between $\mathrm{C} 5$ and C6 (Fig. 1) using our UBC multimechanism SCI apparatus as described previously. ${ }^{6,19,29}$ For contusion, the spinal cord between C5 and C6 was exposed by laminectomy. Custom vertebral clamps held the spinal column rigidly at the transverse processes on a stereotaxic surgical frame. An electromagnetic linear actuator applied a small pre-load to the surface of the dura mater $(0.03 \mathrm{~N})$ with a custom 2-mm-diameter tip. The injury was produced by retracting the actuator $6 \mathrm{~mm}$ above the dura mater and accelerating it into the spinal cord to a maximum displacement of $1.6 \mathrm{~mm}$ (Fig. 1b).

For dislocation and distraction, the animals' posterior ligaments between C5 and C6 were transected, and a C5/6 facetectomy was performed. Custom vertebral clamps were used to securely hold 


\section{a. Normal}

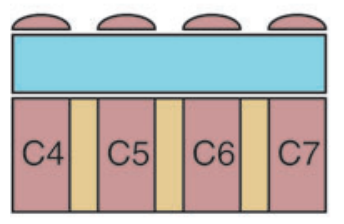

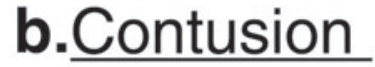

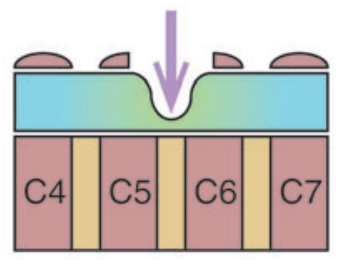

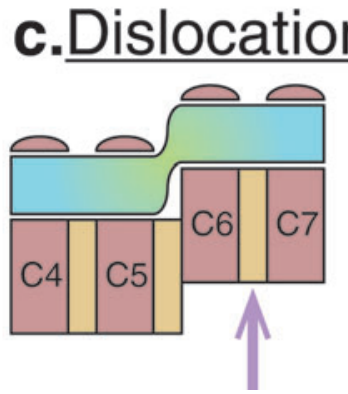

d.Distraction

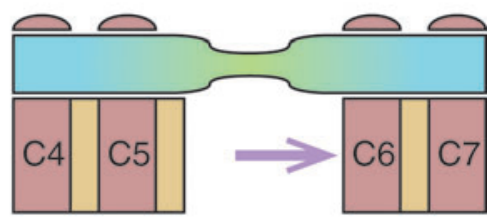

FIG. 1. Schematic diagrams of the three spinal cord injury (SCI) mechanisms. The spinal column orientation for (a) the normal animal and (b-d) the three SCI mechanisms is shown. To produce contusion injury, external displacement was applied to the dorsal spinal cord between C5 and C6 by an actuator with a 2-mm-diameter tip after laminectomy. To produce dislocation injury, external displacement was applied to the transverse processes at $\mathrm{C} 6$ and $\mathrm{C} 7$ dorsal to $\mathrm{C} 5$ by the actuator with vertebral clamps after facetectomy. To produce distraction injury, external displacement was applied to the transverse processes at C6 and C7 caudal to C5 by the actuator with the vertebral clamps after facetectomy.

the transverse processes at $\mathrm{C} 4-\mathrm{C} 5$ on the stereotaxic frame and at $\mathrm{C} 6-\mathrm{C} 7$ on the actuator, respectively. The actuator then acutely translated C6C7 relative to $\mathrm{C} 4-\mathrm{C} 5$, either dorsally by $1.8 \mathrm{~mm}$ (to simulate anteroposterior dislocation), or caudally by $5.6 \mathrm{~mm}$ (to simulate distraction) (Fig. 1c, 1d). An initial pre-load of $2 \mathrm{~N}$ was applied to the spinal column for both the dislocation and distraction injury mechanisms.

The peak displacements were intended to produce a moderate SCI, based on preliminary testing under the same ethics protocol. To maintain some consistency in the severity of injury across different mechanism groups, the peak velocity for all injuries was intended to be approximately $1 \mathrm{~m} / \mathrm{sec}$. This velocity was deemed to be clinically relevant, based on previous work in our laboratory and that of others. ${ }^{30-32}$ Most of the surgical procedures and tools have been described in more detail in our acute studies. ${ }^{6,19,29}$ Since the dislocation and distraction injuries ruptured the C5/6 intervertebral discs, a custom fixation device was applied to the $\mathrm{C} 5$ and $\mathrm{C} 6$ vertebrae to provide mechanical rigidity to the spinal column after inducing the SCI. ${ }^{33}$

Post-injury, the musculature and skin of each animal was sutured, and the animal was placed in an incubator $\left(37^{\circ} \mathrm{C}\right)$ until regaining consciousness. Two doses of buprenorphine $(0.05 \mathrm{mg} / \mathrm{kg}$, SC) and Ringer lactate $(10 \mathrm{~mL}, \mathrm{SC})$ were given to each animal daily, and it was caged individually with oats bedding for the first 2 days post-injury. Ringer lactate was still injected twice a day to animals that exhibited a tendency toward dehydration. The animals were hand-fed and their bladders were manually expressed when needed. Two contusion-, five dislocation-, and five distractioninjured animals reached the humane end-point within 1 week postinjury because of severe weight loss, porphyrin, respiratory distress, and/or paleness. The remainder of the animals ( $n=6$ in each injury) performed the behavioral tasks over the next 8 weeks post-injury.

\section{Histology}

The animals weighed an average of $340 \pm 38 \mathrm{~g}$ at 8 weeks postinjury, immediately before perfusion. These animals and the controls were euthanized by an overdose of 5\% chloral hydrate (intraperitoneal injection). They were perfused with fixative solution (4\% paraformaldehyde in phosphate buffered saline), and the spinal cords were harvested and post-fixed overnight in fixative solution. After several changes of sucrose $(12 \%, 18 \%$, and $24 \%)$ every $24 \mathrm{~h}$, the spinal cords were frozen in optimal cutting temperature compound. They were cut every $0.02 \mathrm{~mm}$ in the cross-sectional plane and divided equally into 10 sets of sections.

One set of spinal cord sections was stained with Luxol fast blue (LFB). One set was blocked with normal donkey serum (1:10, Jackson ImmunoResearch Laboratories, West Grove, PA) and triple stained with primary antibodies against neurofilament $\mathrm{H}$ (NF) (rabbit host, 1:1000, AbD Serotec, Raleigh, NC), neuronal class III $\beta$-tubulin (Tub) (rabbit host, 1:1000, Covance, Princeton, NJ), and myelin basic protein (MBP) (mouse host, 1:20, Millipore, Billerica, MA) in combination with secondary antibodies conjugated with DyLight 594 (donkey host, rabbit antigen, 1:200, Jackson ImmunoResearch Laboratories) and DyLight 488 (donkey host, mouse antigen, 1:400, Jackson ImmunoResearch Laboratories).

One set was blocked with the same normal donkey serum and then stained with primary antibodies against NeuN (mouse host, $1: 200$, Millipore) in combination with secondary antibodies conjugated with DyLight 594 (donkey host, mouse antigen, 1:200, Jackson ImmunoResearch Laboratories) and green fluorescent Nissl (Fluoro Nissl) (1:200, Molecular Probes, Eugene, OR). Each set of sections was stained at the same time and under the same conditions to avoid procedural variation. Standard staining procedures were followed as described previously. ${ }^{34,35}$

The LFB-stained sections were examined under a light microscope (Axioplan 2, Carl Zeiss, Jena, Germany) (5x, NA 0.15) while the NF/Tub/MBP-stained sections and the NeuN/Fluoro Nisslstained sections were examined under a fluorescence microscope (Axio Observer Z1 inverted confocal, Carl Zeiss) (myelinated axons: Plan-Apochromat 63x, NA 1.4, dorsal horn cells: Plan-Apochromat 20x, NA 0.8, ventral horn cells: Plan-Apochromat 10x, NA 0.45). Brightness and contrast were kept constant for all images within each set of sections.

The cross-sectional area of spared tissue (blue-labeled pixels that were darker than the background, representing myelin), the density of myelinated axons (NF/Tub/MBP staining), and the number of surviving cells (NeuN/Fluoro Nissl staining) were measured in the regions of interest (ROIs) in these sections (Fig. 2). In addition, the cross-sectional area of spinal cords, including the anterior median fissure and central canal, was outlined and measured from the LFBstained sections. The central lesion cavity in the LFB-stained sections was also outlined to calculate the volume and rostrocaudal extent of the lesion. Sections at certain distances from the epicenter were selected from each set of spinal cords for analysis (see Fig. 5, 6, 9 for the specific locations).

\section{Data processing and statistical analysis}

Histological analysis (i.e., outlining/segmenting areas, placing ROIs, and counting myelinated axons and cells) and behavioral analysis (i.e., assigning scores and labels) were performed in Photoshop (Adobe Systems, San Jose, CA), Zen (Zeiss), Picture Motion Browser (Sony, Tokyo, Japan), and CatWalk (Noldus Information Technology). All analyses were conducted by observers blinded to the SCI mechanism. Calculations were performed in Excel (Microsoft, Redmond, WA) and MATLAB (MathWorks, Natick, MA). Statistical analysis was performed using SPSS (IBM, Chicago, IL). 


\section{a Spared Tissue (LFB)}

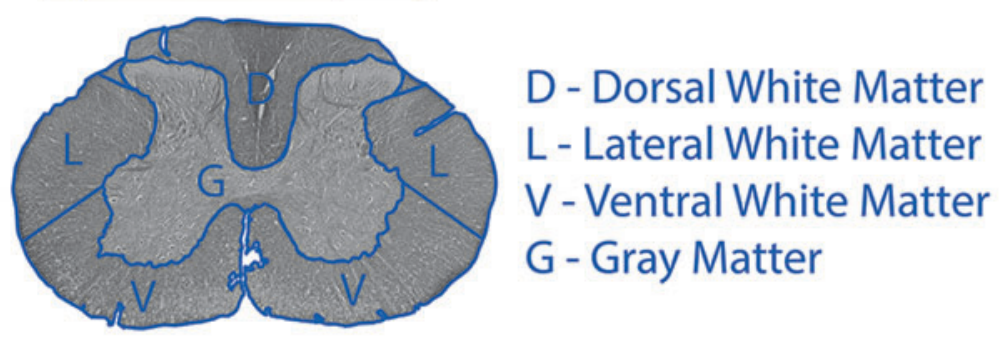

\section{b Myelinated Axons (NF/Tub/MBP)}

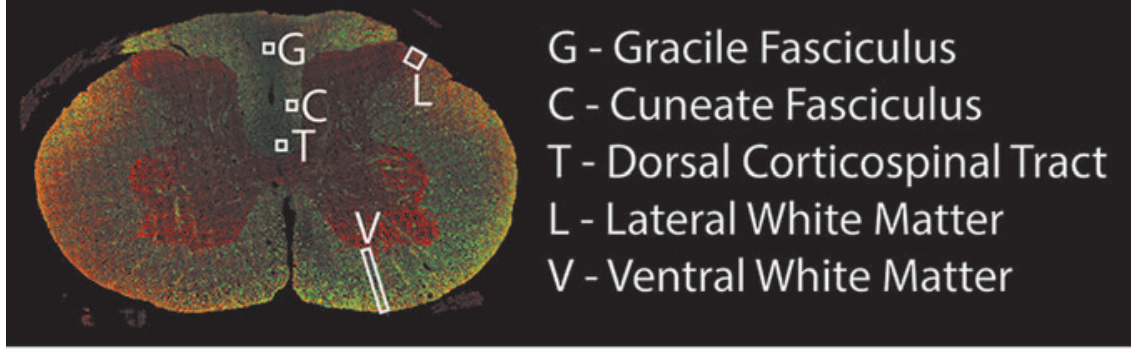

\section{c Surviving Cells (NeuN/FluoroNissl)}

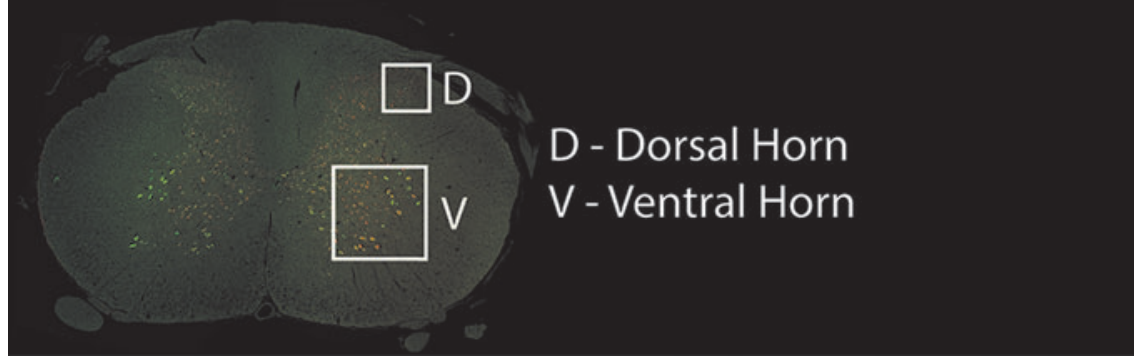

FIG. 2. Histological regions of interest. (a) To measure area of spared tissue in Luxol fast blue (LFB)-stained sections, the dorsal, lateral, and ventral white matter and the gray matter were segmented. The lateral white matter was separated from the dorsal and ventral white matter by drawing straight lines along the lateral sides of the dorsal and ventral horns. (b) To calculate density of myelinated axons in neurofilament $\mathrm{H} / \beta$-tubulin/myelin basic protein-stained sections, several boxes $(65 \mu \mathrm{m} \times 65 \mu \mathrm{m})$ were placed. One box was positioned in the center of the gracile fasciculus. Another box was positioned in the middle right of the dorsal corticospinal tract. A third box was positioned in the right cuneate fasciculus $50 \mu \mathrm{m}$ away from the gracile fasciculus and the dorsal corticospinal tract. A fourth box $(100 \mu \mathrm{m} \times 100 \mu \mathrm{m})$ was positioned in the right lateral white matter next to the dorsal horn. One box $(100 \mu \mathrm{m}$ wide) was positioned in the right ventral white matter medial from the ventral nerve root. The size and position of the boxes could be adjusted to avoid large artifacts and horizontal axons. (c) To count the number of surviving cells in NeuN/Fluoro Nissl-stained sections, images were taken in the right dorsal horn $(20 \mathrm{x})$ and the right ventral horn $(10 \mathrm{x})$ with their sides aligned with the edges of the horns.

Data were not normally distributed (Shapiro-Wilk test, $p>0.05$ ), thus necessitating nonparametric statistical methods to be used. Given that our main research question was on the differences between injury mechanisms, we used the one-way nonparametric analysis of variance (ANOVA) Kruskal-Wallis test at locations along the spinal cord for the histopathologic data and at times postinjury for the behavioral data. We followed each ANOVA with the Mann-Whitney $U$ test (two-tailed, $p<0.05$ ), where appropriate.

Functional improvement across post-injury time points was analyzed using the Friedman test $(p<0.05)$. Given that this approach yielded many statistical comparisons, we have listed those in the Figure legends rather than in the text. To facilitate reading of the results, statements of "less, lower, smaller, did not improve or more, higher, greater, improved" indicate that the difference was statistically significant $(p<0.05)$.

The existence of linear relationships between the histological and behavioral outcomes was assessed by Pearson correlation analysis $(p<0.05)$. For these analyses, the number of myelinated axons (area of spared tissue $\times$ density of myelinated axons) in the dorsal, lateral, and ventral white matter between $5 \mathrm{~mm}$ rostral and caudal, the number of dorsal neurons between $1 \mathrm{~mm}$ rostral and $0.6 \mathrm{~mm}$ caudal, and the number of ventral motoneurons between $2.2 \mathrm{~mm}$ rostral and $1.6 \mathrm{~mm}$ caudal were averaged and used as histological outcome measures. These ranges were used because this is where histological differences existed across the three SCI mechanisms with respect to the epicenter.

\section{Results}

\section{Biomechanical variables}

The measured kinematics and kinetics of the impact injuries are outlined in Table 1 . The peak displacements were the only independent variable in this group. The maximum peak forces were 
Table 1. Summary Biomechanical Data FOR THE Three INJURy Mechanisms

Contusion Dislocation Distraction Normal

\begin{tabular}{lcccc}
\hline Pre-load (N) & 0.03 & 2.0 & 2.0 & - \\
$\begin{array}{l}\text { Maximum } \\
\quad \text { displacement (mm) }\end{array}$ & $1.59 \pm 0.01$ & $1.84 \pm 0.02$ & $5.64 \pm 0.10$ & - \\
$\begin{array}{l}\text { Maximum velocity } \\
\quad(\mathrm{mm} / \mathrm{sec})\end{array}$ & $1206 \pm 78$ & $870 \pm 11$ & $1298 \pm 40$ & - \\
$\begin{array}{l}\text { Maximum force (N) } \\
\text { Maximu }\end{array}$ & $2.57 \pm 0.53$ & $18.0 \pm 1.9$ & $52.2 \pm 2.9$ & -
\end{tabular}

Preload, maximum displacement, maximum velocity, and maximum forces during the contusion $(n=8)$, dislocation $(n=11)$, and distraction $(n=11)$ spinal cord injuries. Pre-load was applied to the cords between C5 and $\mathrm{C} 6$ by the contusion indenter and to the transverse processes at $\mathrm{C} 6$ and C7 by the dislocation/distraction clamps before inducing the actual injuries. All data are mean \pm standard deviation. There were also normal spinal cords that did not receive any injury $(n=5)$.

different across the three SCI mechanisms (Table 1). Note that the higher peak forces in the distraction and dislocation mechanisms were because of the rupturing of the intervertebral discs and other soft tissues during the impact. There was also a somewhat lower peak velocity in the dislocation group compared with the two others.

\section{Spinal cord and spared tissue areas (LFB staining)}

The rostrocaudal extent of the lesion cavities for contusion and dislocation injuries was confined within $3 \mathrm{~mm}$ of the epicenter, while for distraction injuries, the lesion cavity extended farther and was still discernible at $5 \mathrm{~mm}$ rostral and caudal to the epicenter in some animals (Fig. 3). The median (interquartile range) lesion volumes were $2.1(1.7) \mathrm{mm}^{3}$ for contusion, $2.6(1.3) \mathrm{mm}^{3}$ for dislocation, and $3.9(5.0) \mathrm{mm}^{3}$ for distraction, and these were not statistically different, mainly because of large variation in the distraction injuries.

Geometrical change of the spinal cord was observed after both contusion injury and dislocation injury (Fig. 3), with the total spinal cord cross-sectional areas being less than the control animals at and around the epicenter (Fig. 4a). The cross-sectional areas after dislocation were less than after contusion, rostral to the injury site. In contrast, the cross-sectional area of distraction-injured cords was larger than after both contusion and dislocation (Fig. 3, 4a), but was not different from controls.

The cross-sectional areas of spared white matter after contusion and dislocation injuries were lower than in the normal and distracted spinal cords (Fig. 4b). The spared white matter after dislocation was less than after contusion injury at some locations ( $5 \mathrm{~mm}$ rostral, $1.6 \mathrm{~mm}$ rostral, and $0.2 \mathrm{~mm}$ rostral to the epicenter), and these findings were mainly because of large differences in the lateral columns (Fig. 4c). In the dorsal and ventral regions, there were no differences in the area of spared white matter between dislocation and contusion mechanisms surrounding the epicenter, with both having less spared tissue compared with distraction or the control animals (dorsal Fig. 4d, ventral Fig. 4e).

Of interest was that the spared dorsal white matter area after distraction was larger than control, while the lateral white matter area was less than control, leading to there being no overall differences from control after the distraction injury mechanism (Fig. 4c, 4d). This oddity is further addressed in our higher resolution analysis using myelin and axon immunostainings.

The area of spared gray matter tissue was lowest for both the dislocation and contusion injuries compared with controls (between $1 \mathrm{~mm}$ rostral and $3 \mathrm{~mm}$ caudal to epicenter) (Fig. 4f). The gray matter area after distraction injury was smaller than control near the epicenter.

\section{Myelinated axon density (NF/Tub/MBP immunohistochemistry)}

The density of myelinated axons differed across the three SCI mechanisms and depended on the location within the spinal cord. In the gracile fasciculus, the density of myelinated axons was zero after contusion injury at the epicenter and close to zero rostral to the injury site (Fig. 5a). There was a low, but variable, number of surviving (some possibly newly sprouted) myelinated axons after

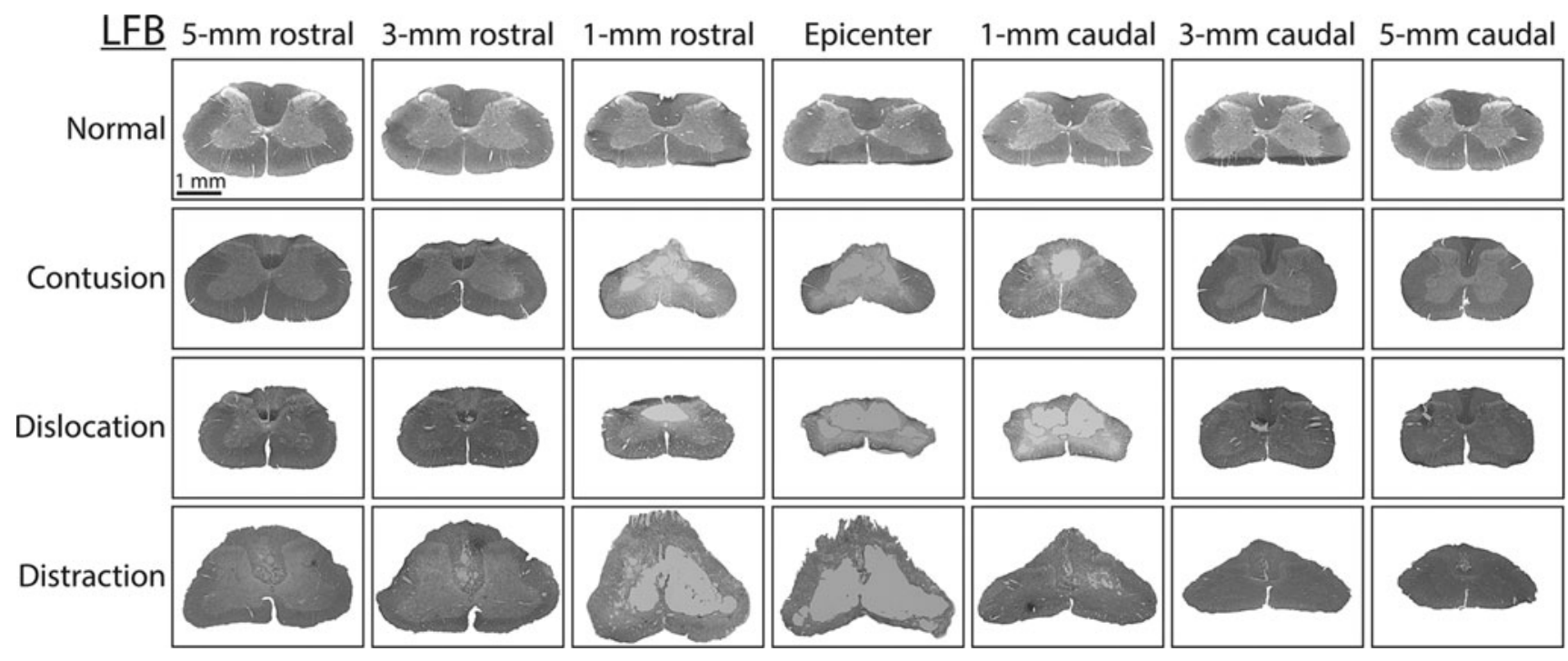

FIG. 3. Representative Luxol fast blue (LFB)-stained sections from normal and injured spinal cords. Representative images are shown of LFB-stained sections for the three injury mechanisms and for a normal spinal cord. The atrophy in the contusion and dislocationinjured cords compared with the control cords should be noted. The distraction cord areas were noticeably larger than both contusion and dislocation injured cords. The lesion cavity in both contusion and dislocation was confined to the epicenter and $1.6 \mathrm{~mm}$ rostrally and caudally, while the lesion extended as much as $5 \mathrm{~mm}$ after distraction injury, particularly in the caudal direction. 
dislocation injury and consistent survival of few myelinated axons after distraction injury in all locations-both being lower than control (except for dislocation at $3 \mathrm{~mm}$ and $5 \mathrm{~mm}$ caudal).

The pattern of damage between the three mechanisms qualitatively differed at the location of the gracile fasciculus (Fig. 6a). Because of the impact imparted on the dorsal surface of the spinal cord in the contusion injury model, the gracile fasciculus was destroyed and replaced by cavitation at the epicenter. The ascending sensory fibers degenerated rostrally, leaving behind some myelin debris and axons that likely represent sprouted (or displaced) sensory afferents from the adjacent cuneate fasciculus. The degree of damage and cavitation was slightly less in the gracile fasciculus after dislocation injury, which variably destroyed most axons at the epicenter and rostral to it, leaving extensive myelin debris in its wake. In both contusion and dislocation injury models, the proximal part of the most ascending sensory axons remained preserved at 3 and $5 \mathrm{~mm}$ caudal to injury, and partial sparing is seen as close as $1 \mathrm{~mm}$ from the epicenter after dislocation but not after contusion (Fig. 5a). Interestingly the extent and distribution of the damage differed after distraction injury, which left in its wake a reduced packing density of myelinated axons that were often separated by enlarged extracellular spaces (Fig. 6a).

Similar patterns of myelinated axon damage across the mechanisms were observed at the cuneate fasciculus where the density of myelinated axons was lower than control for the contusion (between $5 \mathrm{~mm}$ rostral and $5 \mathrm{~mm}$ caudal to epicenter), dislocation (between $5 \mathrm{~mm}$ rostral and $3 \mathrm{~mm}$ caudal to epicenter), and the distraction mechanism (between $5 \mathrm{~mm}$ rostral and $0 \mathrm{~mm}$ and also $5 \mathrm{~mm}$ caudal to epicenter).

Widespread axonal damage of the dorsal corticospinal tract (CST) was observed across the three SCI mechanisms (Fig. 5b, 6b). There were zero axons $\pm 1 \mathrm{~mm}$ to the epicenter after contusion, close to zero axons after dislocation, and reduced density with some sparing of myelinated axons at all distances from the epicenter after distraction injury (Fig. 5b). There were fewer myelinated axons at $3 \mathrm{~mm}$ caudal and $\pm 5 \mathrm{~mm}$ from the epicenter after contusion compared with dislocation. All three injury models spared about 30 $40 \%$ of the myelinated corticospinal axons at $5 \mathrm{~mm}$ proximal to the injury, leaving a mixture of myelin debris (i.e., green dots in Fig. 6b) and numerous demyelinated corticospinal axons (i.e., red dots without surrounding green in Fig. 6b) in its wake.
Both contusion and dislocation caused large cavitation that interrupted the CST in and around the lesion epicenter (Fig. 6b). The area of the distal CST 3 and $5 \mathrm{~mm}$ caudal to the dislocation and contusion injuries contained extensive myelin debris and axons that were larger than normal corticospinal axons and likely represent displaced ascending or sprouting sensory fibers. In contrast, after distraction, a large number of axons, consistent with the typically small corticospinal axon morphology, was still preserved at all levels studied, although the degree of myelin coverage appeared highly variable, suggesting extensive demyelination and/or selective sparing of unmyelinated axons.

In the lateral white matter, there was a lower density of myelinated axons at the epicenter after the distraction injury compared with contusion injury (Fig. 5c). We observed large numbers of small myelinated and unmyelinated axons (apparently spared or possibly demyelinated) at all levels post-contusion injury in the lateral white matter (Fig. 7a). In contrast, after dislocation, numerous axons were devoid of myelin and likely demyelinated based on their large caliber. Although the number of myelinated axons encountered in the lateral white matter after distraction was low at the lesion epicenter, there was an abundance of unmyelinated and apparently demyelinated axons (based on their large caliber).

The density of myelinated axons in the ventral white matter for all three SCI mechanisms was lower than in the controls at the epicenter and $1 \mathrm{~mm}$ caudally (Fig. 5d). In addition, the density was lower than control at $1 \mathrm{~mm}$ rostral to the epicenter for dislocation injury. The density of myelinated axons after contusion was less than in control and distraction-injured animals at all caudal locations. Qualitatively, the normal histology of the ventral column is dominated by very large myelinated axons (likely vestibulospinal) that are surrounded by numerous smaller fibers (Fig. 7b). All three SCI mechanisms produced an extensive degeneration of these large caliber axons in and around $( \pm 1 \mathrm{~mm})$ the lesion epicenters, leaving large myelin debris in the wake (green onion-like layered bodies).

\section{Neuron survival (NeuN/Fluoro Nissl immunohistochemistry)}

The number of surviving neurons differed across the three SCI mechanisms and also varied between different locations in the gray

FIG. 4. The total spinal cord area and areas of spared tissue from the Luxol fast blue (LFB)-stained sections. Cross-sectional area of (a) total spinal cord, (b) spared tissue of the total white matter, (c) spared tissue of the lateral white matter, (d) spared tissue of the dorsal white matter, (e) spared tissue of the ventral white matter, and (f) spared tissue of the gray matter for the different SCI mechanisms $(n=6)$ and the normal $(n=5)$ as quantified from LFB-stained sections. Values for spared tissue analysis were obtained by measuring the number of pixels that were darker than the background intensity. All data are presented as median with quartiles and offset horizontally for clarity. The position along the spinal cord extends from $-5 \mathrm{~mm}$ (rostral) through $+5 \mathrm{~mm}$ (caudal) from the epicenter $(0 \mathrm{~mm})$. All statistical differences reported are $p<0.05$. Statistical results in (a): (i) differences from normal: contusion -5 to $+1 \mathrm{~mm},+5 \mathrm{~mm}$; dislocation -5 to $+5 \mathrm{~mm}$; distraction none; (ii) differences between contusion and dislocation: $-5,-3,-1.6,-0.2 \mathrm{~mm}$; (iii) differences between contusion and distraction: $-3 \mathrm{mo}+2.2 \mathrm{~mm}$; (iv) differences between dislocation and distraction: -5 to $+3 \mathrm{~mm}$. Statistical results in (b): (i) differences from normal: contusion -1 to $+1 \mathrm{~mm}$; dislocation -3 , to $+1 \mathrm{~mm}$; distraction none; (ii) differences between contusion and dislocation: $-5,-1.6,-0.2 \mathrm{~mm}$; (iii) differences between contusion and distraction: -3 to $+1.6 \mathrm{~mm}$; (iv) differences between dislocation and distraction: -3 to $+1.6 \mathrm{~mm}$. Statistical results in (c): (i) differences from normal: contusion $-1,+3 \mathrm{~mm}$; dislocation -3 to $+3 \mathrm{~mm}$; distraction $0,+3 \mathrm{~mm}$; (ii) differences between contusion and dislocation: -2.2 to $+1 \mathrm{~mm}$; (iii) differences between contusion and distraction: none; (iv) differences between dislocation and distraction: -1.6 to $+1 \mathrm{~mm}$. Statistical results in (d): (i) differences from normal: contusion -1 to $+1 \mathrm{~mm}$; dislocation $-3,-1,0 \mathrm{~mm}$; distraction -5 to $+5 \mathrm{~mm}$; (ii) differences between contusion and dislocation: $+5 \mathrm{~mm}$; (iii) differences between contusion and distraction: -5 to $+5 \mathrm{~mm}$; (iv) differences between dislocation and distraction: -5 to $+5 \mathrm{~mm}$. Statistical results in (e): (i) differences from normal: contusion $0,+1 \mathrm{~mm}$; dislocation $0 \mathrm{~mm}$; distraction $+1 \mathrm{~mm}$; (ii) differences between contusion and dislocation: $-5 \mathrm{~mm}$; (iii) differences between contusion and distraction: -1 to $+0.6 \mathrm{~mm},+3 \mathrm{~mm}$; (iv) differences between dislocation and distraction: -1 to $+0.6 \mathrm{~mm}$. Statistical results in (f): (i) differences from normal: contusion -1 to $+3 \mathrm{~mm}$; dislocation $-5 \mathrm{~mm},-1$ to $+3 \mathrm{~mm}$; distraction $0,+1 \mathrm{~mm}$; (ii) differences between contusion and dislocation: $-5 \mathrm{~mm}$; (iii) differences between contusion and distraction: -2.2 to $+2.2 \mathrm{~mm}$; (iv) differences between dislocation and distraction: -5 to $+2.2 \mathrm{~mm}$. 
matter. The neuron number in the dorsal horn was lower in the contusion and dislocation-injured spinal cords than in the control or distraction-injured cords at and around the epicenter (Fig. 8a). The number of neurons in the dorsal horn was similar to controls in the distraction-injured cords. The changes in neuron numbers are also appreciated in the micrographs (Fig. 9a), where parts of the dorsal horn were destroyed at the epicenter and turned into lesion cavity after contusion and dislocation injuries. In the dislocation model, this extensive damage extended even farther to at least $1 \mathrm{~mm}$ caudal to the epicenter. a
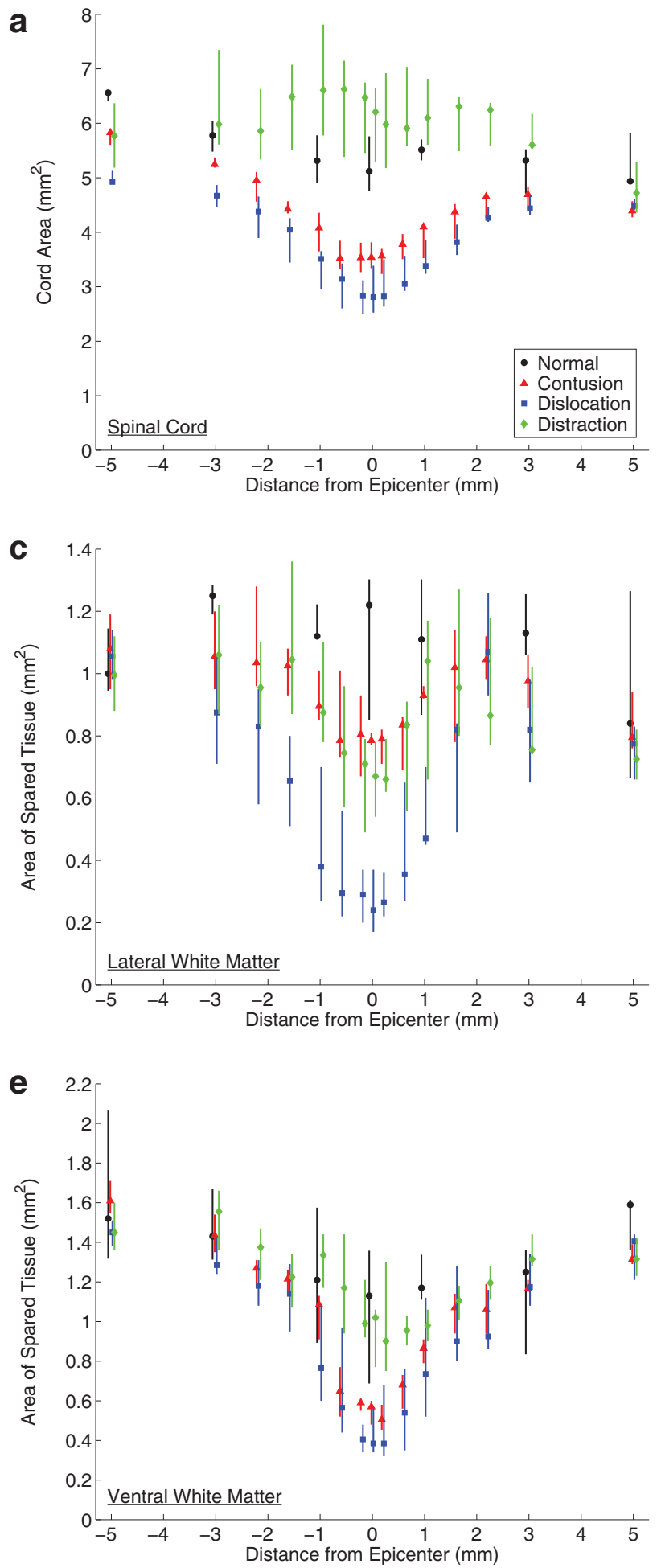
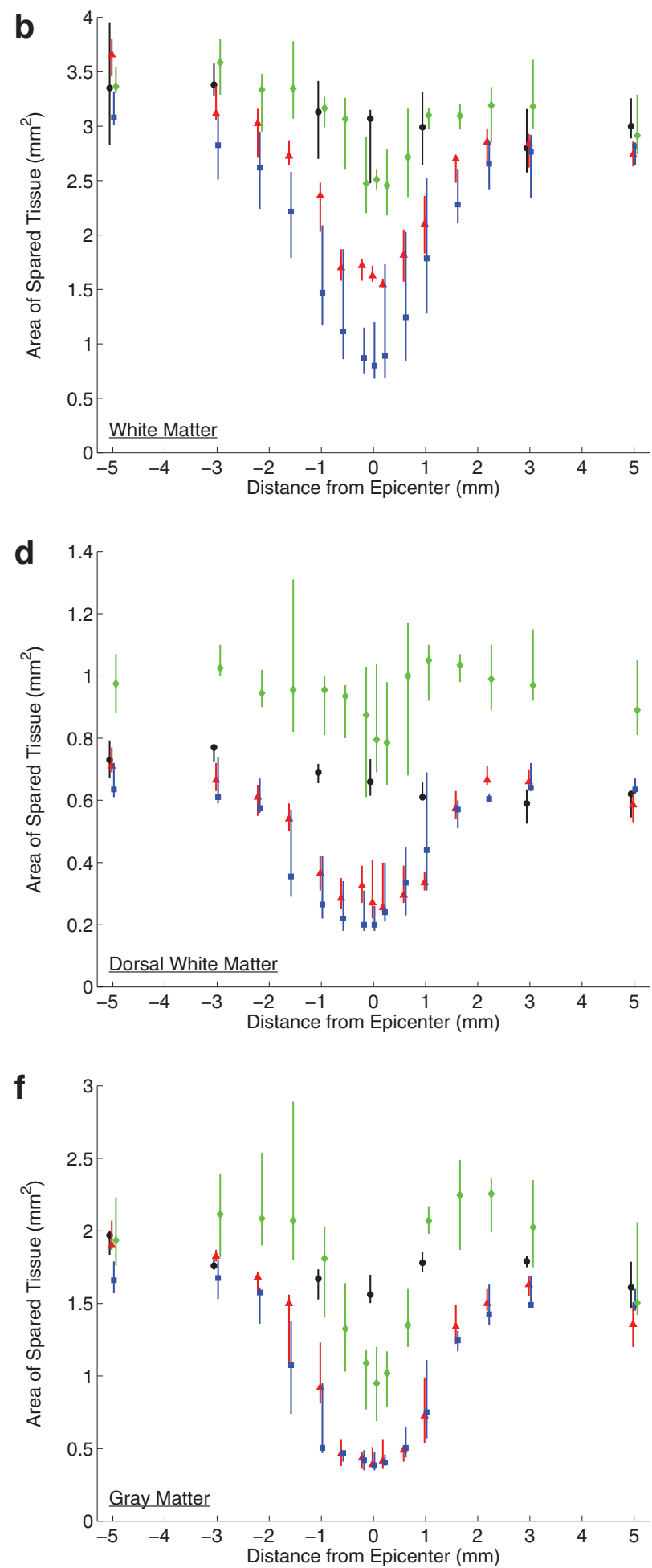

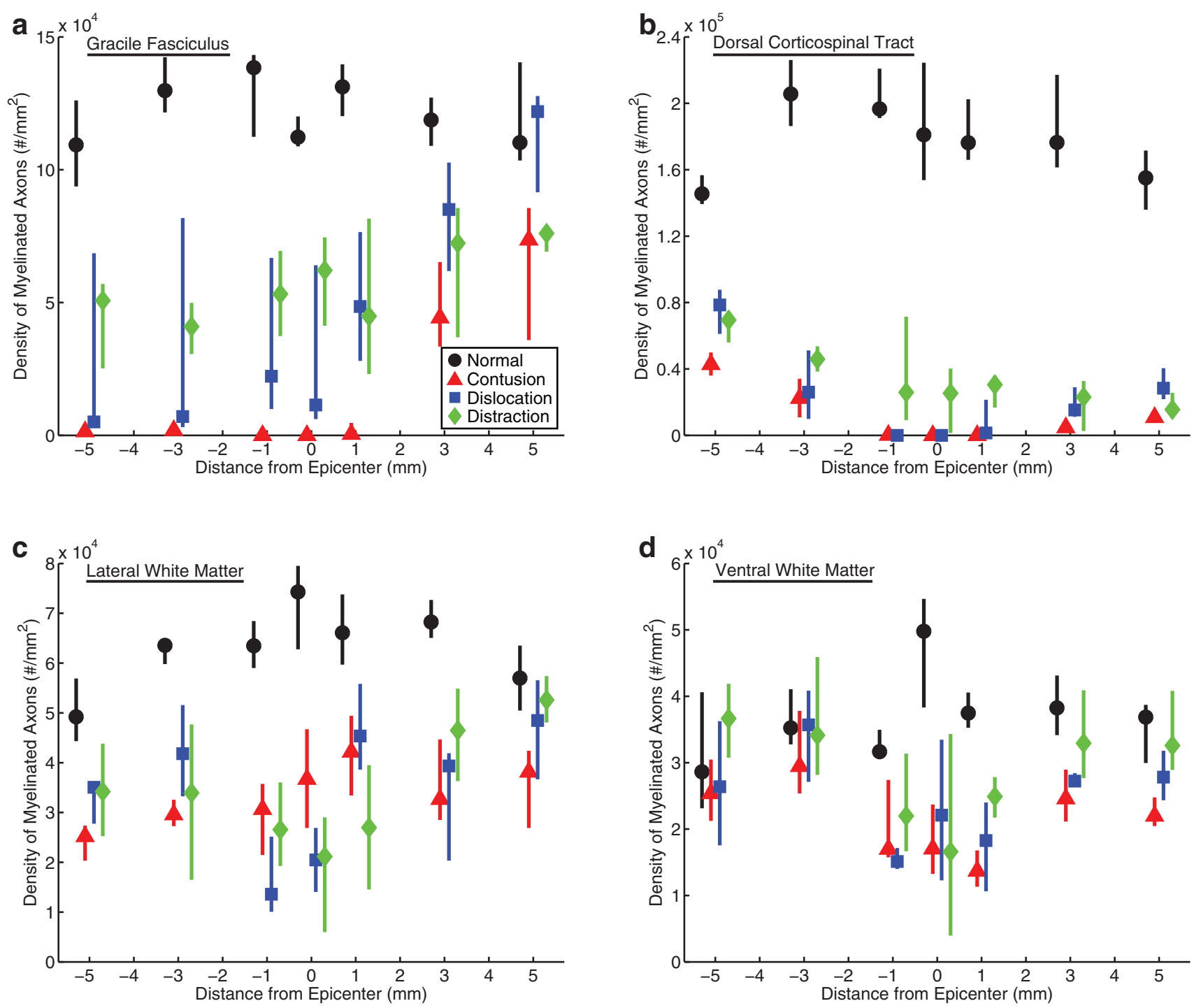

FIG. 5. The density of myelinated axons for the different spinal cord injury mechanisms. All data are presented as median with quartiles and offset horizontally for clarity. The position along the spinal cord extends from $-5 \mathrm{~mm}$ (rostral) through $+5 \mathrm{~mm}$ (caudal) from the epicenter $(0 \mathrm{~mm}$ ). All statistical differences reported are $p<0.05$. (a) Gracile fasciculus statistical results: (i) differences from normal: contusion -5 to $+5 \mathrm{~mm}$; dislocation -5 , to $+1 \mathrm{~mm}$; distraction -5 to $+5 \mathrm{~mm}$; (ii) differences between contusion and dislocation: -5 to $+1,+5 \mathrm{~mm}$; (iii) differences between contusion and distraction: $-5,-1,0,+1 \mathrm{~mm}$; (iv) differences between dislocation and distraction: $+5 \mathrm{~mm}$. (b) Dorsal corticospinal tract statistical results: (i) differences from normal: contusion -5 to $+5 \mathrm{~mm}$; dislocation -5 to $+5 \mathrm{~mm}$; distraction -5 to $+5 \mathrm{~mm}$; (ii) differences between contusion and dislocation: $-5,+3,+5 \mathrm{~mm}$; (iii) differences between contusion and distraction: -5 to $+1 \mathrm{~mm}$; (iv) differences between dislocation and distraction: -1 to $+1 \mathrm{~mm}$. (c) Lateral white matter statistical results: (i) differences from normal: contusion -5 to $+5 \mathrm{~mm}$; dislocation -5 to $0 \mathrm{~mm},+3 \mathrm{~mm}$; distraction -1 to $+3 \mathrm{~mm}$; (ii) differences between contusion and dislocation: $-5 \mathrm{~mm}$; (iii) differences between contusion and distraction: $0,+5 \mathrm{~mm}$; (iv) differences between dislocation and distraction: none. (d) Ventral white matter statistical results: (i) differences from normal: contusion 0 to +5 mm; dislocation $-1,0,+1 \mathrm{~mm}$; distraction $0,+1 \mathrm{~mm}$; (ii) differences between contusion and dislocation: none; (iii) differences between contusion and distraction: $-5,+1$ to $+5 \mathrm{~mm}$; (iv) differences between dislocation and distraction: none.

The number of surviving motoneurons (large NeuN/Fluoro Nissl positive neurons) in the ventral horn was less than in controls in all three SCI mechanisms at and around the epicenter (Fig. 8b). The absence of motoneurons at the epicenter and at $1 \mathrm{~mm}$ caudal to it after dislocation is shown in Fig. 9b, while some neurons were consistently preserved here after contusion injuries. Interestingly, after distraction injury, the number of surviving motoneurons was highly variable, and between $1.6 \mathrm{~mm}$ rostral and caudal to the lesion, some animals were devoid of ventral horn neurons over several millimeters (Fig. 8b).

\section{Behavioral/functional outcomes}

The ladder rung walking test and CatWalk results are not presented; too many injured animals (one in contusion and four in dislocation) could not perform these tests after the injuries, because of their inability to grab onto the ladder rungs or step with weightsupport on the Plexiglas surface of the Catwalk.

We observed differences across the three SCI mechanisms in the various post-injury behavioral tests. In the Martinez locomotor rating scale, the forelimb score for all animals decreased at 1 week 


\section{a}

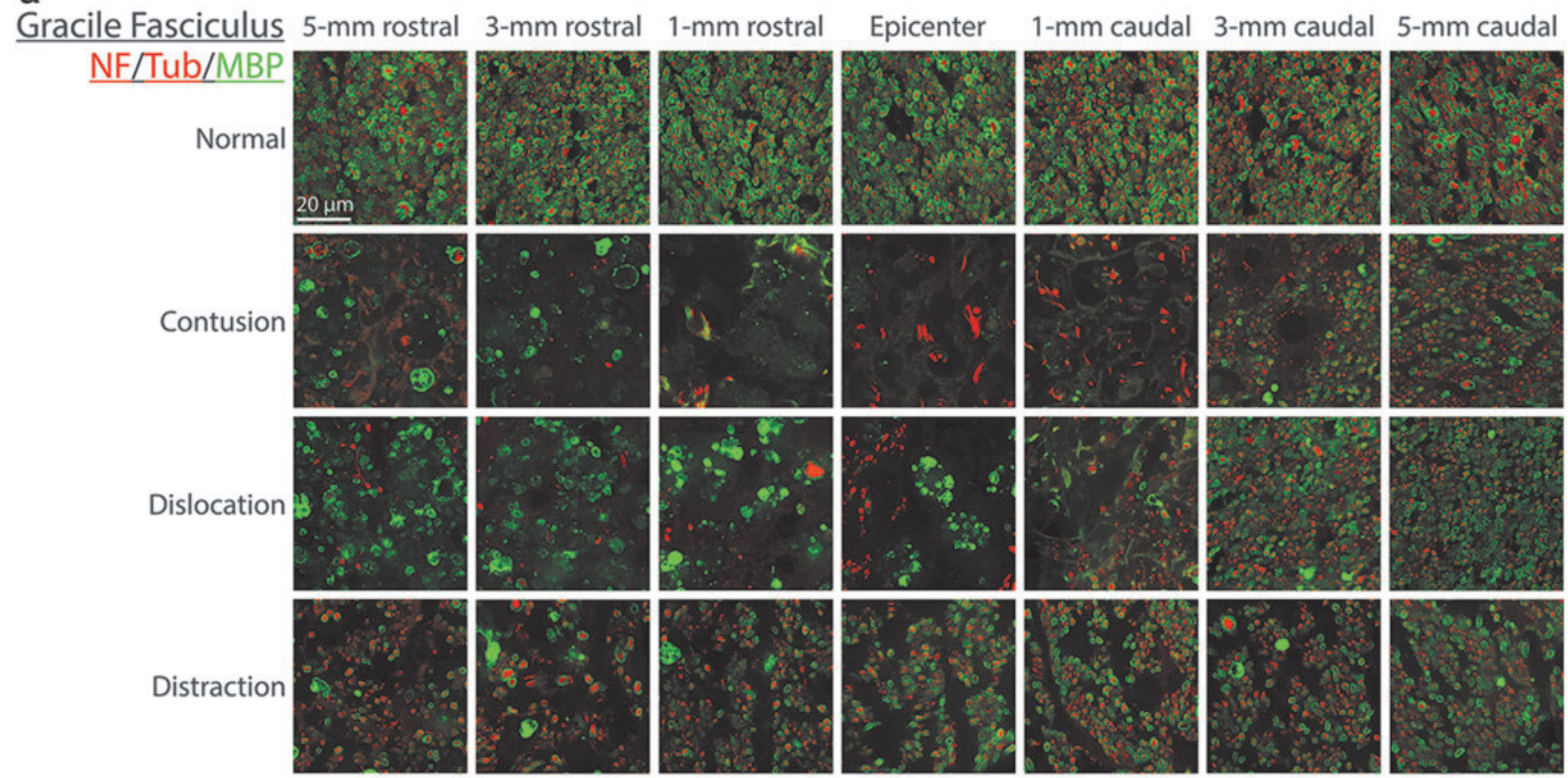

b

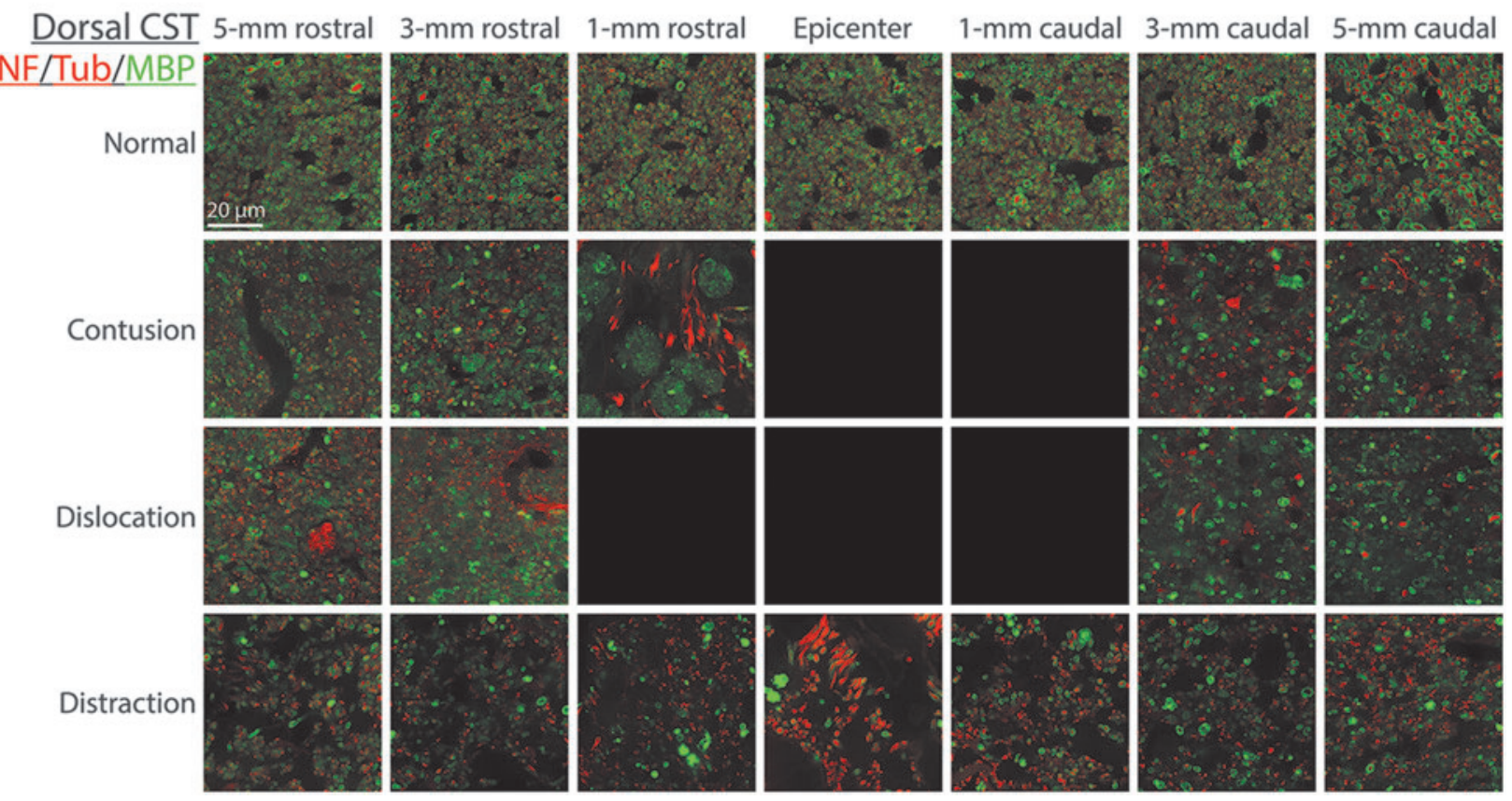

FIG. 6. Micrographs of myelinated axons in the (a) gracile fasciculus and (b) dorsal corticospinal tract (CST). Representative confocal micrographs of the regions of interest used in the quantitative analysis of white matter damage immunostained for neurofilament $\mathrm{H} / \beta$ tubulin for axons (in red) and myelin basic protein for myelin/myelin debris in green. The normal control tracts show a dense packing of small myelinated axons in the gracile fasciculus (GF) and even smaller ones in the dorsal CST. These intact fibers display the typical green myelin rings surrounding a red axon in the middle. Contusion and (somewhat less so) dislocation injuries destroy both tracts from the epicenter distally, leaving in the wake of Wallerian degeneration myelin debris (green dots) and a few large axons (displaced or sprouted). Distal is rostral in the ascending GF and caudal in the descending CST. The damage proximal to the epicenter is indicative of some dieback in both tracts. The damage after distraction is less focal in the GF showing a gradual thinning of this ascending tract from caudal to rostral and appearance of a few larger profiles in the rostral segments that are likely displaced axons from the cuneate fascile (entering the spinal cord rostral to injury). The CST shows a loss of the typical small myelinated axons at all levels with some partial preservation rostrally. The apparent preservation of the CST in the caudal segments is from displaced/sprouted larger sensory axons. Scale bar $20 \mu \mathrm{m}$. MBP, myelin basic protein. 


\section{a}

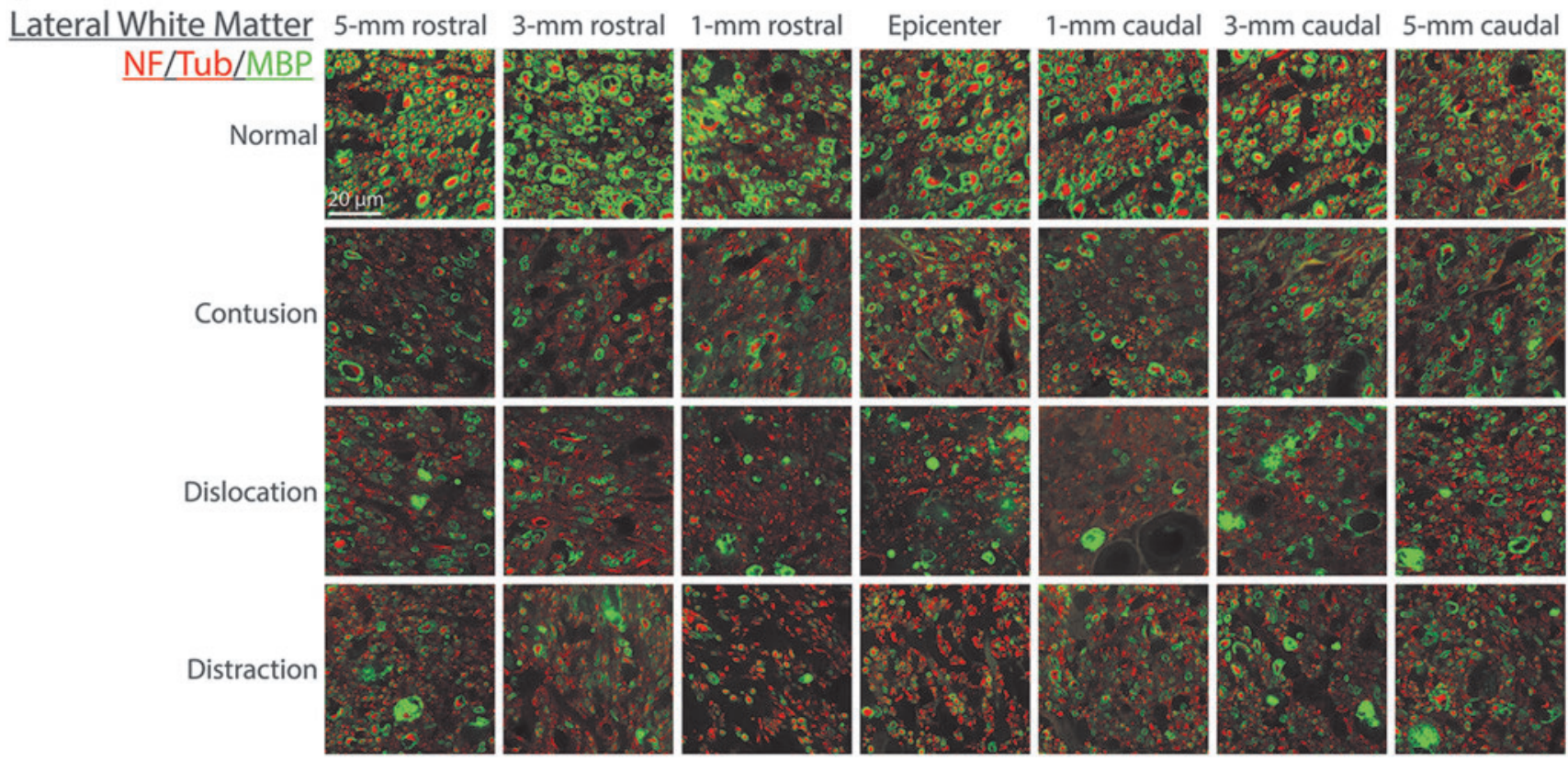

\section{b}

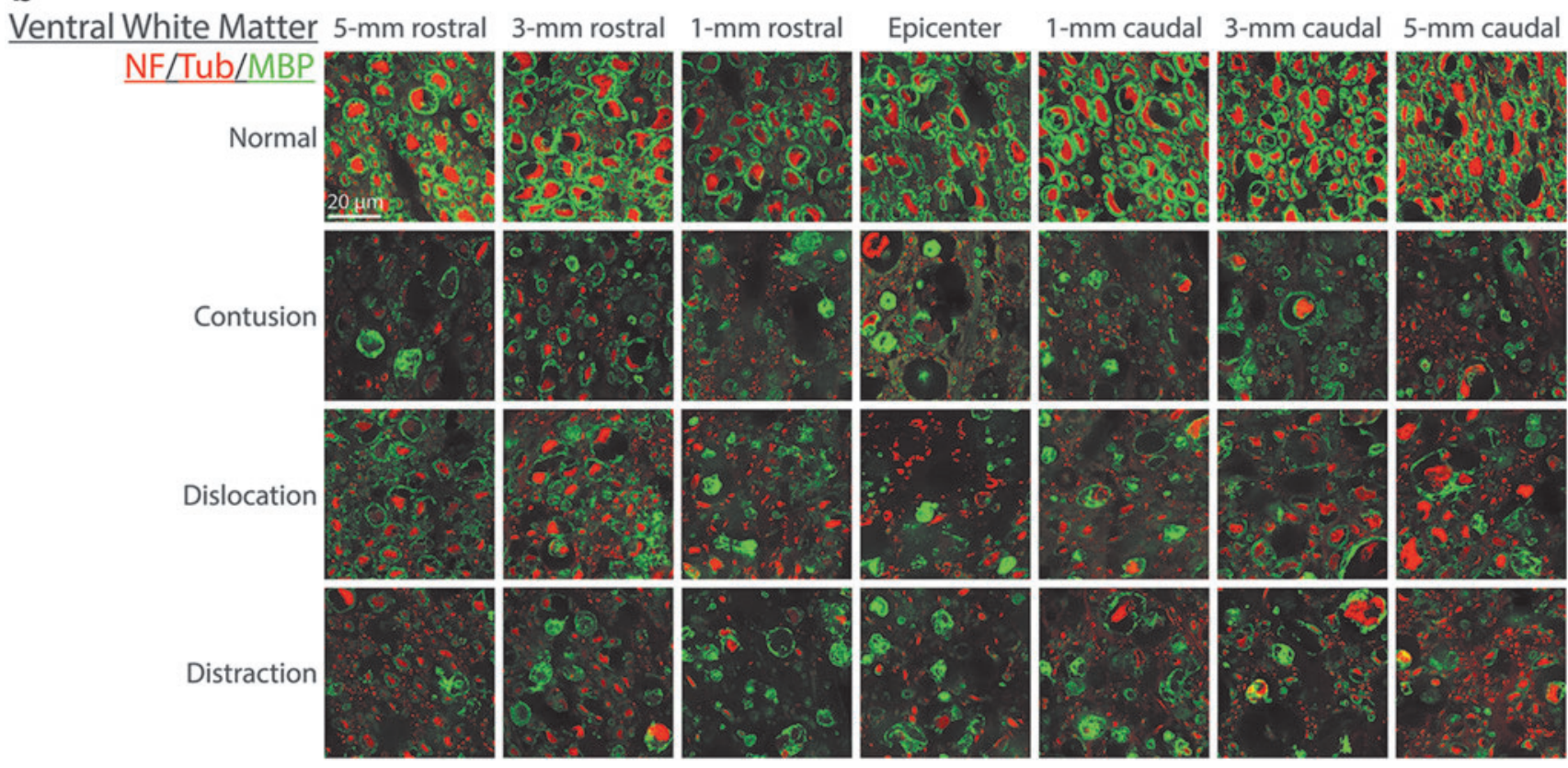

FIG. 7. Micrographs of myelinated axons in the (a) lateral white matter and (b) ventral white matter. Representative confocal micrographs of the regions of interest used in the quantitative analysis of white matter damage immunostained for neurofilament $\mathrm{H} / \beta$ tubulin (NF/Tub) for axons (in red) and myelin basic protein for myelin/myelin debris in green. The normal control tracts show a dense packing of predominantly midsize myelinated axons in the lateral white matter and predominantly medium to large ones in the ventral white matter. These intact fibers display the typical green myelin rings surrounding a red axon in the middle. All three injury types produce a thinning of the myelinated axon packing in the lateral white matter at all levels reflecting the damage of ascending and descending fibers while the damage of the ventral white matter is very pronounced at the injury epicenter and further distally (with some preservation rostrally). The damage of the lateral white matter is somewhat more pronounced after dislocation than in the other two models. Note the abundance of green dots or empty rings representing myelin debris and of red dots without green rings most likely showing demyelinated (and possibly sprouted) axons; they appear particularly prominent in the lateral white matter around the epicenter after distraction injury. Scale bar $20 \mu \mathrm{m}$. MBP, myelin basic protein. 

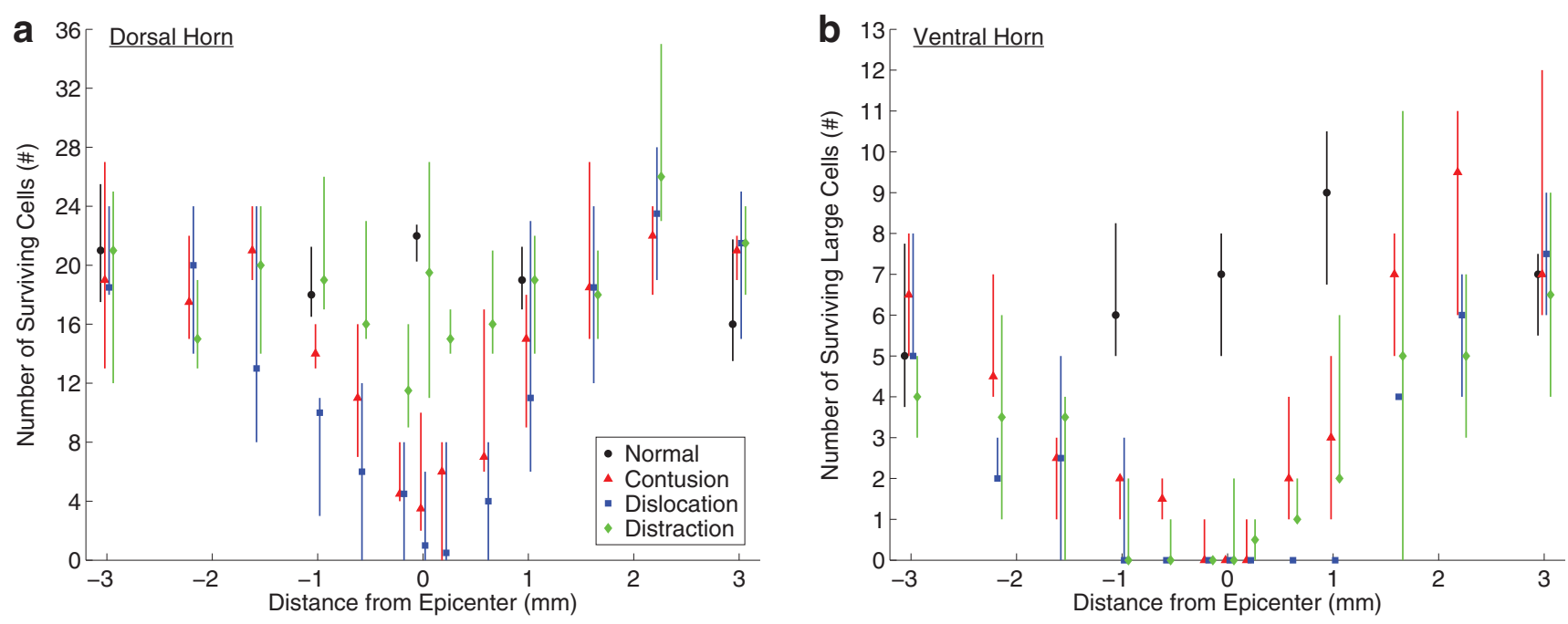

FIG. 8. The number of surviving neurons for the different spinal cord injury mechanisms. Data are presented as median with quartiles and offset horizontally for clarity. The position along the spinal cord extends only from $-3 \mathrm{~mm}$ (rostral) through $+3 \mathrm{~mm}$ (caudal) from the epicenter $(0 \mathrm{~mm})$. All statistical differences reported are $p<0.05$. (a) Number of surviving cells in the dorsal horn statistical results: (i) differences from normal: contusion $0 \mathrm{~mm}$; dislocation $-1,0 \mathrm{~mm}$; distraction none; (ii) differences between contusion and dislocation: $-1 \mathrm{~mm}$; (iii) differences between contusion and distraction: $-0.2,0,+0.2 \mathrm{~mm}$; (iv) differences between dislocation and distraction: -1 to $+0.6 \mathrm{~mm}$. (b) Number of surviving large cells in the ventral horn statistical results (a cell was determined to be large if it was larger than a typical dorsal cell): (i) differences from normal: contusion $-1,0,+1 \mathrm{~mm}$; dislocation $-1,0,+1 \mathrm{~mm}$; distraction -1 , $0 \mathrm{~mm}$; (ii) differences between contusion and dislocation: $-2.2,-0.6,+0.6,+1,+1.6 \mathrm{~mm}$; (iii) differences between contusion and distraction: none; (iv) differences between dislocation and distraction: $+0.6,+1 \mathrm{~mm}$.

post-injury (Fig. 10a). After that time, the contusion and distraction animals improved up to 8 weeks post-injury and reached levels similar to those before SCI, while the dislocation animals did not improve beyond 1 week. As expected, after the incomplete cervical injuries used in our models, the impairments in hindlimb function as measured with this scale were of a transient nature in rats, but the hindlimb score did not improve after all three SCIs from 1-8 weeks post-injury, and the animals remained below preinjury score (Fig. 10b). The total score decreased at 1 week postinjury and then improved to 8 weeks (Fig. 10c), but the animals for all three SCI mechanisms still had lower total scores at 8 weeks post-injury than pre-injury, mainly because of impairment of the forelimb movements. The FLAS mirrored the results of the Martinez total score (Fig. 10d).

In the grooming test, the contusion- and distraction-injured animals dropped after injury, although there was considerable variability after distraction at week 1 and then scores improved toward week 8 (Fig. 10e). By 8 weeks, the majority of the animals in the contusion and distraction groups were able to reach as far up as the ears (i.e., score 4). In contrast, grooming scores in the dislocation-injured animals decreased after injury and did not improve up to week 8 , with lower scores than both contusion and distraction between 2 and 8 weeks. The majority of dislocationinjured animals were only able to touch the snout at 8 weeks postSCI (score of 1-2).
In the grip strength test, a dramatically different pattern was observed. Baseline grip strength before injury averaged around $400 \mathrm{~g}$ of force for all three groups. The contusion- and dislocationinjured animals exhibited lower strength at week 2 post-injury and then improved to baseline or slightly higher values by week 8 , possibly because of increased spasticity (Fig. 10f). Grip strength in the distraction-injured animals, however, decreased at week 2 , but did not improve thereafter up to week 8 , and recovered between 2-8 weeks post-injury to only $50 \%$ of pre-injury baseline values.

In the Montoya staircase, the number of pellets taken was lower than before injury in the contusion-injured animals between weeks 2 and 6 post-injury, in the dislocation-injured animals at week 6 post-injury, and in the distraction-injured animals between weeks 2 and 3 and between weeks 6 and 8 post-injury. The number of pellets eaten was lower than at pre-injury baseline in the contusion-injured animals between weeks 2 and 6 post-injury, in the dislocation-injured animals between weeks 6 and 8 , and in the distraction-injured animals between weeks 2 and 8 post-injury. No improvement was observed after all three SCIs, and no differences were observed between groups.

\section{Histology and behavior correlations}

Scatterplots between the various histological outcomes and the behavioral scores are displayed in Figure 11, and many significant

FIG. 9. Micrographs of surviving cells in the (a) dorsal horn and (b) ventral horn. Representative micrographs of the regions of interest used in the quantitative analysis of neurons immunostained for NeuN/Fluoro Nissl. Contusion and even more pronounced dislocation lead to a loss of dorsal horn neurons as well as dorsal horn tissue around the lesion epicenter, which is partially occupied by cavity in this location. This loss of neurons in the dorsal horn is far less pronounced after distraction injury. In the ventral horn, the loss of large neurons is most pronounced after contusion and dislocation at the epicenter and more widespread after dislocation injury, and some cavitation is still visible $1 \mathrm{~mm}$ caudal to injury here. The number of large ventral horn neurons after distraction is somewhat more variable and in some animals nil rostal to the presumed epicenter. Scale bars $100 \mu \mathrm{m}$ for dorsal horn and $200 \mu \mathrm{m}$ for ventral horn images. 
a

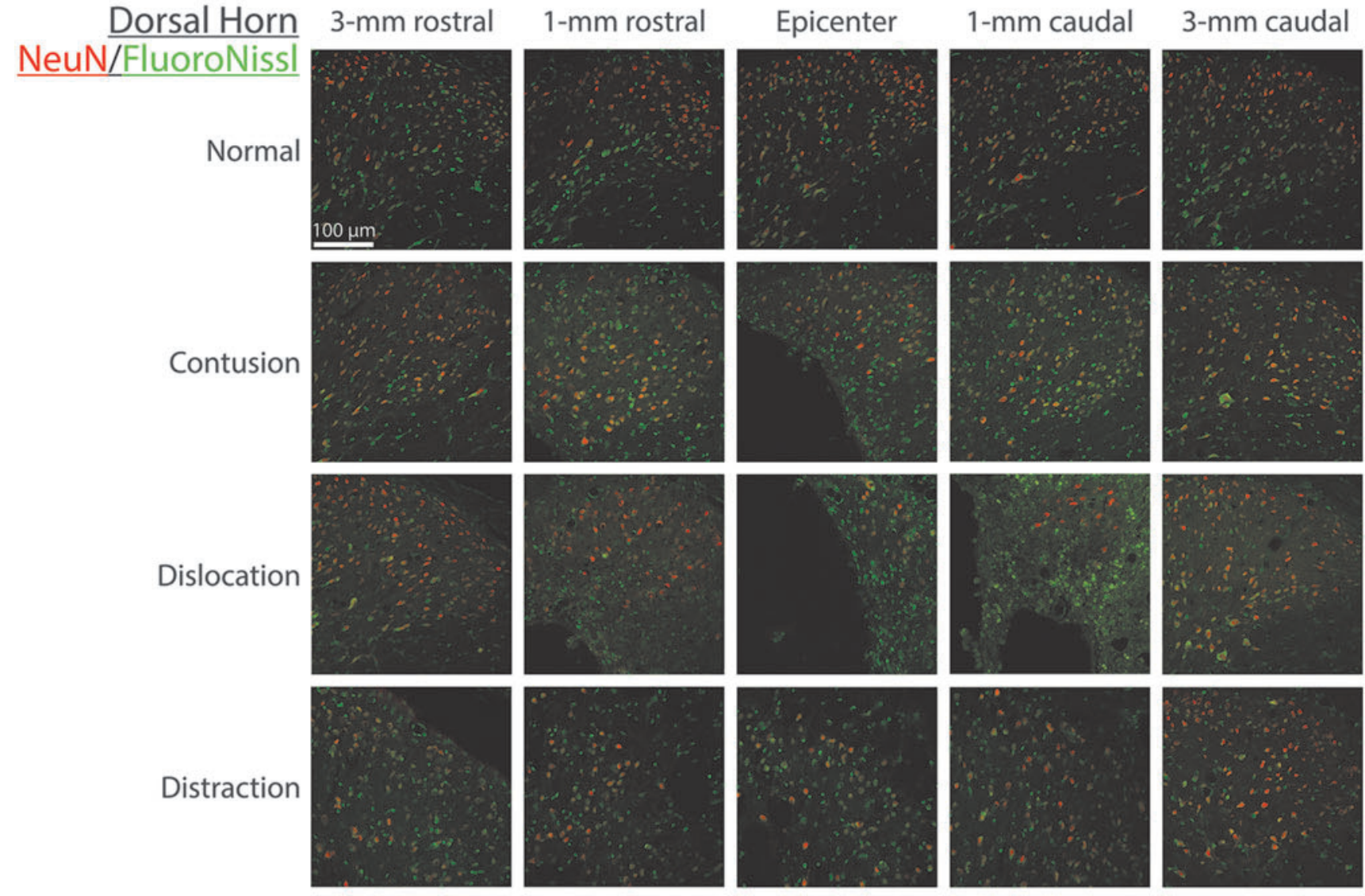

b

Ventral Horn 3-mm rostral

1-mm rostral

NeuN/FluoroNissl

Normal

$200 \mu \mathrm{m}$

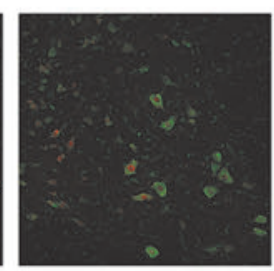

Epicenter

1-mm caudal

3-mm caudal
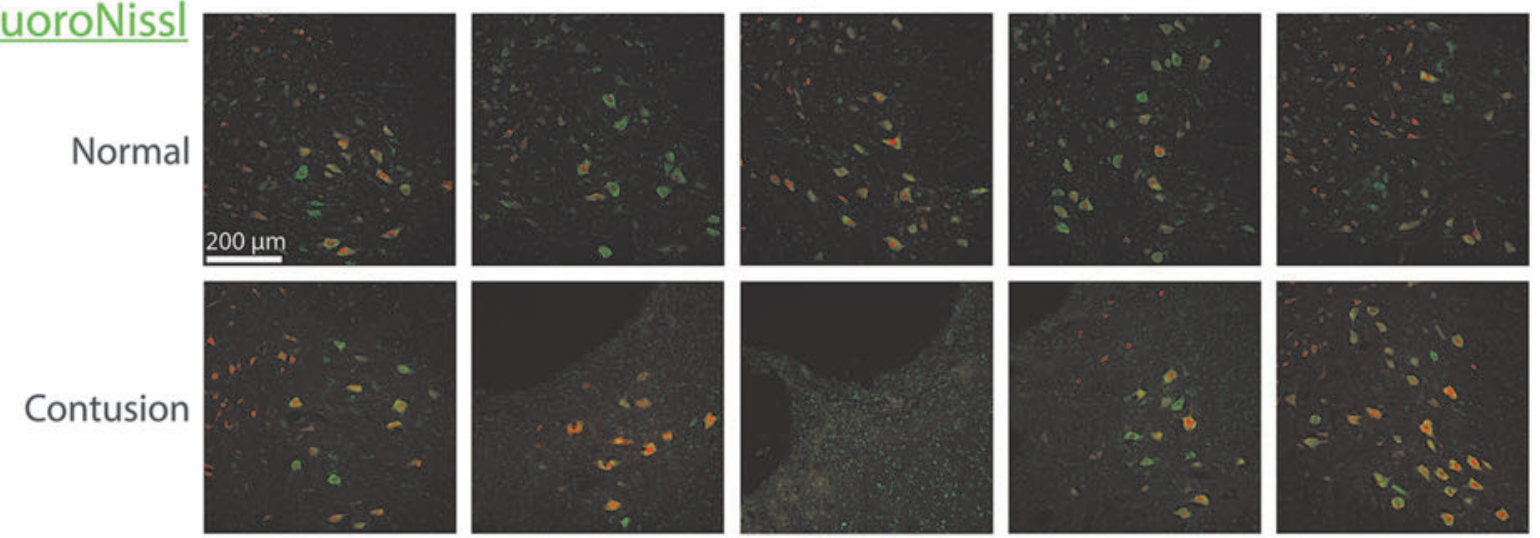

Dislocation
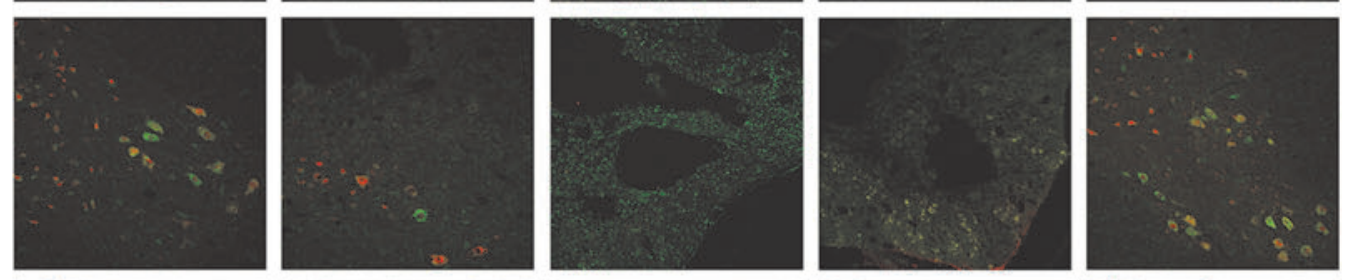

Distraction
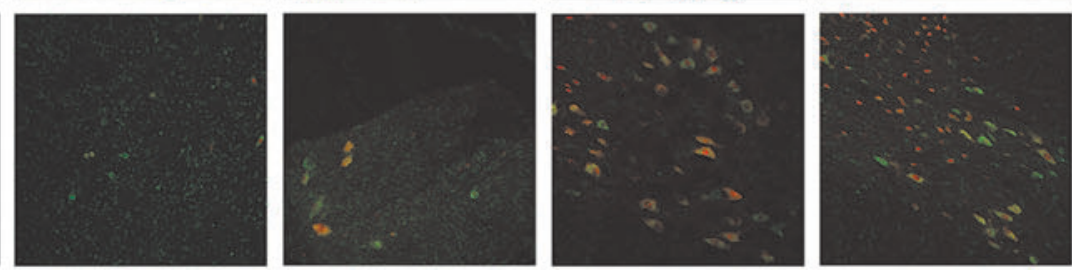
correlations were found. Significant relationships may shed a causal link between the structures in the spinal cord and certain behaviors, but we are cautious to not make too many assertions here, because it is a highly complex system that would likely require a much larger study and a multivariate statistical analysis. A second observation from these plots is the apparent clustering of the data from the three SCI mechanisms in some plots. An example is the grip strength-average number of dorsal cells scatterplot. This may demonstrate how the different SCI mechanisms result in distinct functional injuries.

\section{Discussion}

This study is the first to compare three clinically relevant mechanisms of cervical SCI up to 8 weeks post-injury in a rat model. We observed distinct differences in the histopathology across the three injury mechanisms, and a brief summary is outlined below and shown schematically in Figure 12. Contusion caused the most severe loss of myelinated axons in the dorsal white matter (point A, Fig. 12). Dislocation caused the greatest overall loss of white matter tissue, especially in the lateral white matter (point B), and the rostrocaudal extent of this loss was greatest after dislocation. Dislocation also caused the greatest neuronal cell death in both the ventral and dorsal horns (point $\mathrm{C}$ ), although the differences from contusion were small. Distraction caused substantial structural alteration in the white matter (point D), while sparing the most myelinated axons overall. Distraction also caused the most extensive lesion cavity (point E), particularly in the dorsal white matter. These key findings highlight the complex and varied effect of different spinal cord deformations on histopathology.

Of great interest was that these pathological changes in the spinal cord tissue were associated with behavioral differences in the animals. After a distraction injury, the animals displayed no improvement in grip strength over time in contrast to the animals with contusion or dislocation injuries. Animals injured by distraction and contusion gradually recovered their forelimb function on the locomotor tests. After dislocation, the animals displayed virtually no improvement in the grooming test with time in contrast to the animals with contusion or distraction injury where there was improvement in grooming. Therefore, these results suggest that the SCI mechanism affects both spinal cord histopathology and animal behavior and thus likely contributes to the heterogeneity of SCI in the human population.
The three SCI mechanisms studied herein represent clinically relevant spinal column injuries that produce a range of deformation patterns in the spinal cord. Contusion SCI, reflecting a burst fracture spinal column injury, produces very focal dorsoventral compressive strain to the white and gray matter directly under the impactor and also high tensile lateral strains in these same tissues. ${ }^{36}$ Dislocation SCI, which represents a fracture dislocation spinal column pattern, produces a widespread dorsoventral compressive strain of the spinal cord and axial tensile strain along the length of the C5-C6 intervertebral space. ${ }^{36}$ In this sense, dislocation is a less focally localized injury than contusion. Distraction SCI reflects a component of flexion-distraction or extension-distraction spinal column injuries, which is clearly not as common as burst fracture or fracture-dislocation. Nevertheless, it is a distinctly different type of cord deformation that merits our understanding.

The histological changes at 8 weeks post-injury as described herein are similar to some observations from the early time-point studies at $5 \mathrm{~min}$ and $3 \mathrm{~h}$ post-injury. ${ }^{6,19}$ Most notable is the observation of substantially less spared lateral white matter after the dislocation injury and the increased rostral extent of these differences. The increased rostrocaudal extent of the neuronal damage after dislocation also mirrors the previous findings. ${ }^{6,19}$

Some of the most dramatic differences from the earlier timepoint studies were observed in the distraction animals. As noted earlier, the distraction injuries produced a more diffuse pattern of tissue damage at $5 \mathrm{~min}$ and $3 \mathrm{~h}$ post-SCI than after contusion and dislocation, where there was little apparent hemorrhage and a small degree of plasma membrane compromise. ${ }^{6,19}$ Therefore, it was surprising to see substantial damage to the spinal cord 8 weeks postinjury after the distraction mechanism, characterized by an increased size, a large central lesion volume, and substantial demyelination across all regions of the spinal cord. The reasons for this increase in size are poorly understood and might be related to multiple dehiscence in the tissue created by stretching and multiple failures, leaving in its wake apparent spaces. These may not be apparent early after injury with our methods used, and hence longer term survival is important to assess the effect of various secondary damage mechanisms to the spinal cord. An additional possible reason for the differences with the analysis at $5 \mathrm{~min}$ and $3 \mathrm{~h}$ after distraction is that in the current study, we applied a larger maximum displacement (i.e., $5.6 \mathrm{~mm}$ with no head flexion) than was applied in the earlier studies (i.e.. $4.1 \mathrm{~mm}$ with 15 degrees of head flexion). To evaluate the maximum displacements to be imposed in each mechanism, we

FIG. 10. The behavioral test data for the animals injured by the three spinal cord injury mechanisms. Data are presented as median with quartiles and offset horizontally for clarity. The time goes from -1 week (pre-injury) to +8 weeks (post-injury). All statistical differences reported are $p<0.05$. (a) Martinez locomotor rating scale, forelimb score statistical results: (i) differences from -1 week: contusion +1 to +8 weeks; dislocation +1 to +6 week; distraction +1 to +6 weeks; (ii) differences between contusion and dislocation: none; (iii) differences between contusion and distraction: none; (iv) differences between dislocation and distraction: none. (b) Martinez locomotor rating scale, hindlimb score statistical results: (i) differences from -1 week: contusion +1 to +8 weeks; dislocation +1 to +8 weeks; distraction +1 to +8 weeks; (ii) differences between contusion and dislocation: +8 weeks; (iii) differences between contusion and distraction: none; (iv) differences between dislocation and distraction: +8 weeks. (c) Martinez locomotor rating scale, total score statistical results: (i) differences from -1 week: contusion +1 to +8 weeks; dislocation +1 to +8 weeks; distraction +1 to +8 weeks; (ii) differences between contusion and dislocation: none; (iii) differences between contusion and distraction: none; (iv) differences between dislocation and distraction: none. (d) Forelimb locomotor assessment scale score statistical results: (i) differences from -1 week: contusion +1 to +8 weeks; dislocation +1 to +4 weeks; distraction +1 to +8 weeks; (ii) differences between contusion and dislocation: none; (iii) differences between contusion and distraction: none; (iv) differences between dislocation and distraction: none. (e) Grooming test score statistical results: (i) differences from -1 week: contusion +1 to +8 weeks; dislocation +1 to +8 weeks; distraction +1 to +8 weeks; (ii) differences between contusion and dislocation: +1 to +8 weeks; (iii) differences between contusion and distraction: none; (iv) differences between dislocation and distraction: +2 to +8 weeks. (f) Grip strength test statistical results: (i) differences from -1 week: contusion +2 weeks; dislocation +2 weeks; distraction +2 to +8 weeks; (ii) differences between contusion and dislocation: none; (iii) differences between contusion and distraction: $+3,+6,+8 \mathrm{wk}$; (iv) differences between dislocation and distraction: +3 to +8 weeks. 

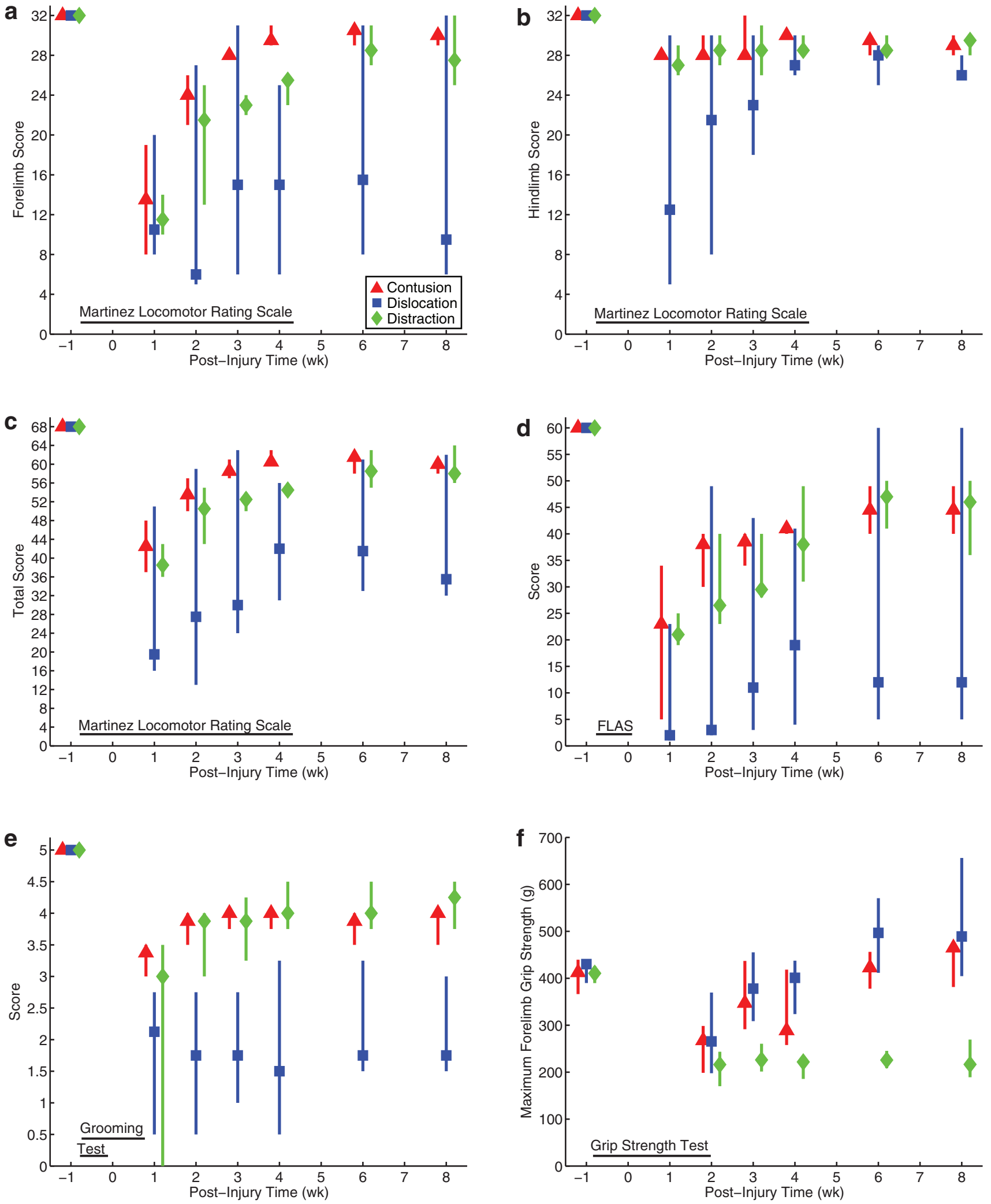

performed pilot studies and found that animals with the $4.1 \mathrm{~mm}$ distraction magnitude without head flexion $(n=5)$ had no noticeable tissue damage and locomotor deficit at week 1 post-injury. Therefore, the displacement magnitude was increased to produce what we assessed as a moderate SCI.

We continue to work on reducing the variability of the injuries produced in the animals after the dislocation and distraction SCI mechanisms. These two mechanisms rely on the integrity of the vertebral clamp-vertebra interface to apply the forces necessary to the spine to produce the respective dislocation or distraction injury. 


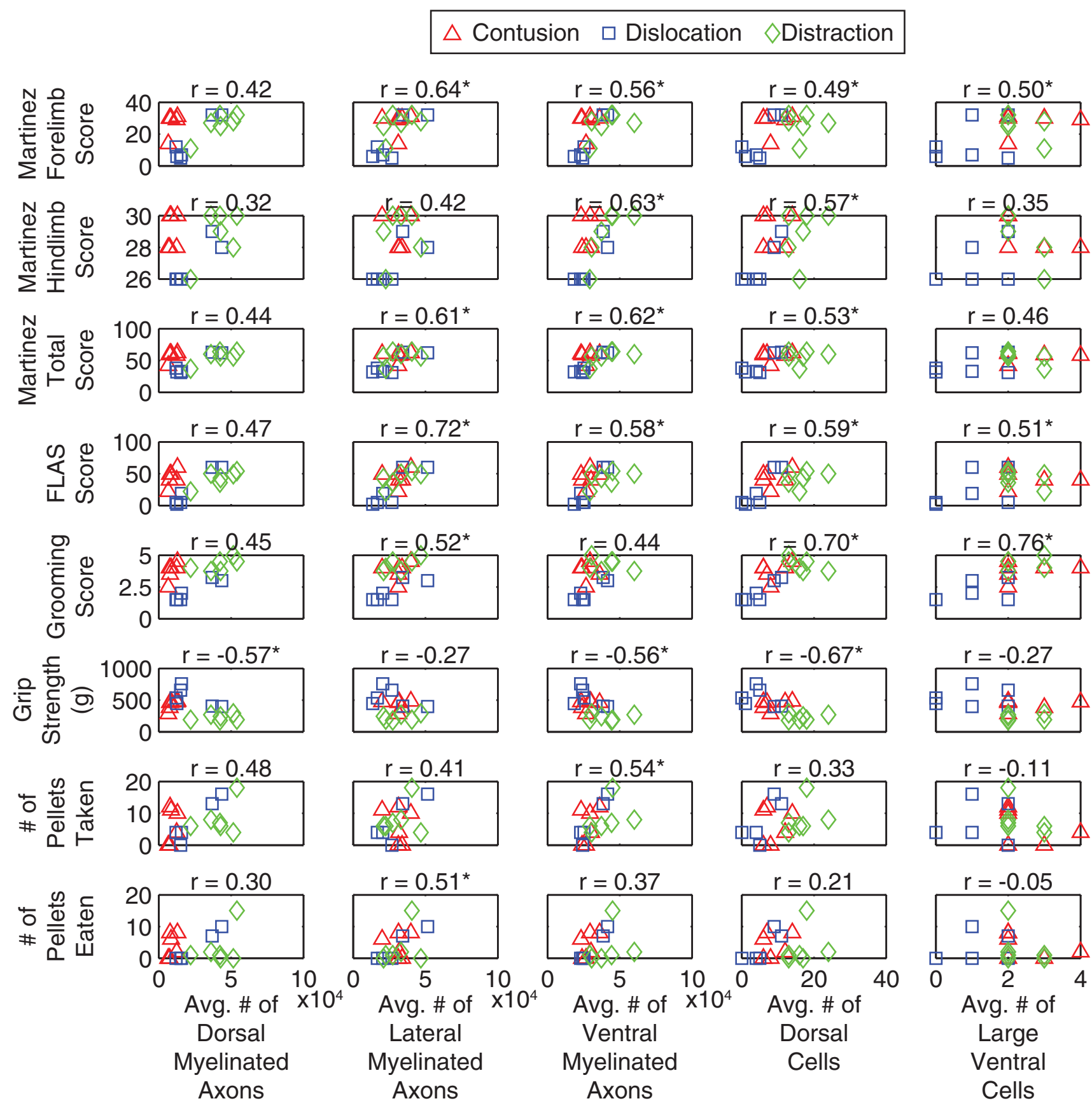

FIG. 11. Scatterplots showing the relation between histological and behavioral outcomes $(n=18$, except in the Montoya staircase, $n=17$ ) evaluated by Pearson correlation coefficient (r). The number of myelinated axons was measured by multiplying the density of myelinated axons by the area of spared tissue in the dorsal, lateral, and ventral white matter. The average of these numbers were then calculated between $5 \mathrm{~mm}$ rostral and caudal to the epicenter. The number of dorsal and large ventral cells was averaged between $1 \mathrm{~mm}$ rostral and $0.6 \mathrm{~mm}$ caudal and between $2.2 \mathrm{~mm}$ rostral and $1.6 \mathrm{~mm}$ caudal to the epicenter, respectively. These cord segments in histology were selectively analyzed because the injury differences were found within these distances relative to the epicenter. Behavioral data were obtained from the Martinez locomotor rating scale, the forelimb locomotor assessment scale, the grooming test, the grip strength test, and the Montoya staircase 8 week post-injury. ${ }^{*}$ Statistically correlated with each other $(p<0.05)$.

For both mechanisms, the intervertebral disc is ruptured during the trauma aimed at producing SCI. In dislocation, the disc is ruptured in pure shear, and this results in forces of approximately $20 \mathrm{~N}$. In distraction, the disc is ruptured in pure tension and forces above $40 \mathrm{~N}$ are typically needed. ${ }^{6}$ When forces of this magnitude are applied to the rat spine, a high stress is transmitted to the clampvertebra interface and thus, there is potential for slippage.
We have recently performed some high-speed radiologic analyses of the dislocation injuries and discovered that the interface remains rigid in some animals, while in others it slips somewhat. ${ }^{37}$ We think that this is the cause of the increased variability that we observe between these two injury mechanisms.

Despite this variability, some intriguing observations were made in the behavioral tests in animals across the different injury 

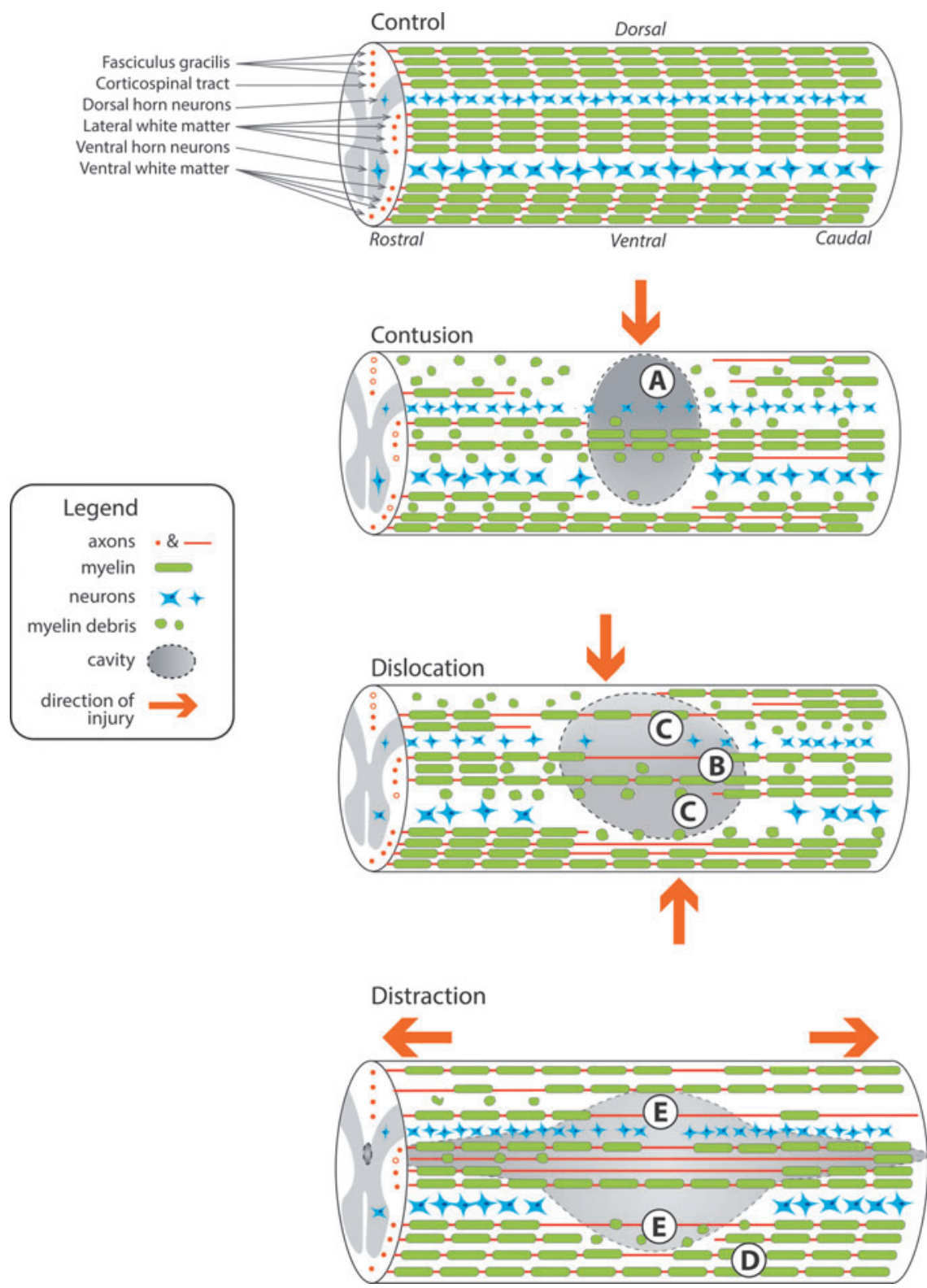

FIG. 12. Schematic diagrams of the anatomic damage patterns in the spinal cord. The damage to the myelinated axons in the dorsal, lateral, and ventral white matter are depicted as is the damage to the neuronal cell bodies in the gray matter. The red arrows show the directions of the mechanical deformations. The severe loss of myelinated axons after contusion can be seen in the dorsal white matter (point A). Dislocation caused the greatest overall loss of white matter tissue, especially in the lateral white matter (point B), and the rostrocaudal extent of this loss was greatest after dislocation. Dislocation also caused the greatest neuronal cell death in both the ventral and dorsal horns (point C), although the differences from contusion were small. Distraction caused substantial structural alteration in the white matter (point D), while sparing the most myelinated axons overall. Distraction also caused the most extensive lesion cavity (point E), particularly in the dorsal white matter. Neuronal survival after distraction injuries was highly variable. The schematic diagram does not accurately reflect the spinal cord areas compared with control or changes from rostral to caudal.

mechanisms. The grooming test is useful in assessing upper limb range of motion and coordination that is involved in grooming. ${ }^{23}$ The severely impaired forelimb grooming function in the dislocationinjured animals was likely related to their severe neuronal cell death between approximately C5 and C6 and severe tissue loss between approximately $\mathrm{C} 4$ and $\mathrm{C} 6 .{ }^{38,39}$ This activity relies on preservation of neuronal networks for movement pattern generation, which possibly explains the lack of recovery in the dislocation-injured animals compared with the contusion-injured animals and in the distraction- injured animals. The high correlation between neural damage and the grooming score (Fig. 11) supports this analysis.

The grip strength was useful in detecting the maximum forelimb grip strength that was different in the distraction-injured animals than in the contusion-injured animals and in the dislocation-injured animals. The severely weakened maximum forelimb grip strength in the distraction-injured animals might be related to their lesion extending further rostrocaudally with tissue cavitation and dehiscence, hence involving a longer segment of the spinal cord than 
after the other two lesions (especially between the caudal end of C5 and the rostral part of T1 for distal forelimb function ${ }^{38,39}$ ).

The Montoya staircase, the ladder rung walking test, and the CatWalk were too difficult for some injured animals to perform. That being said, they still suggested that the dislocation-injured animals had the most difficulty in performing these tasks, and only the distraction-injured animals could all perform these tasks beginning at week 2 post-injury. The expanded Martinez locomotor rating scale, the grooming test, and the grip strength were the most useful discriminative tests in this study-i.e., for the detection of injury mechanism-related differences in behavior.

The differences observed across the three SCI mechanisms provide us with insights that may be helpful for the development and application of future therapeutic approaches. Obviously, each therapy targets a limited number of injury pathomechanisms. ${ }^{40,41}$ Theoretically, therapies that promote axonal growth may be most effective for contusion injury followed by dislocation injury because of the widespread axonal interruptions observed at and around the epicenter in these injury mechanisms. Therapies that promote remyelination may be of greatest value in distraction injuries and less so after dislocations and contusions. Tissue sparing approaches may be most effective for dislocation injuries while still of benefit in the other lesion models.

Ultimately, we seek to understand the heterogeneity behind human SCI. Clearly, we see in humans SCIs occurring with different biomechanical mechanisms and with different injury severities-variability that is clearly not reproduced in most preclinical modeling of SCI. Here, we endeavor to understand how these different biomechanical mechanisms influence outcome.

\section{Conclusion}

Contusion, dislocation, and distraction SCI mechanisms elicit differential effects on histological and behavioral outcomes in a rat model. It is true that the different injury mechanisms in this experimental paradigm likely induce injuries of varying severity as well. It would be ideal to be able to impart the exact same degree of energy to the exact same distribution of the spinal cord despite the different injury mechanisms. Obviously, this is technically challenging to do, given the complexity of inducing what amounts to a "closed" SCI with the distraction and dislocation mechanisms. Nonetheless, even accounting for potential differences in injury severity, the different injury mechanisms resulted in different histopathology in different regions of the spinal cord. Clearly, more research is needed to better characterize these complex injury models.

It is also recognized that human injuries-particularly severe injuries-may occur with some combination of each of these morphologic patterns of injury to the spinal column, and that it is often very difficult to discern precisely how the injury occurred. Given these differences, future SCI research may need to focus on personalized treatments that may emerge for specific SCI patterns.

\section{Acknowledgments}

We wish to acknowledge the technical assistance of Yuan Jiang and Caron Fournier in this research project and Cheryl Niamath for her artistic expertise. We also acknowledge the financial assistance of the Canadian Institutes for Health Research (CIHR) towards this research in the form of a research grant (\# 86645).

\section{Author Disclosure Statement}

No competing financial interests exist.

\section{References}

1. Ackery, A., Tator, C., and Krassioukov, A. (2004). A global perspective on spinal cord injury epidemiology. J. Neurotrauma 21, 1355-1370.

2. Sekhon, L.H., and Fehlings, M.G. (2001). Epidemiology, demographics, and pathophysiology of acute spinal cord injury. Spine (Phila Pa 1976) 26, Suppl 24, S2-S12.

3. Wyndaele, M., and Wyndaele, J.J. (2006). Incidence, prevalence and epidemiology of spinal cord injury: what learns a worldwide literature survey? Spinal Cord 44, 523-529.

4. Fehlings, M.G., and Baptiste, D.C. (2005). Current status of clinical trials for acute spinal cord injury. Injury 36, Suppl 2, B113-B122.

5. Tator, C.H. (2006). Review of treatment trials in human spinal cord injury: issues, difficulties, and recommendations. Neurosurgery 59, 957-987.

6. Choo, A.M., Liu, J., Lam, C.K., Dvorak, M., Tetzlaff, W.. and Oxland, T.R. (2007). Contusion, dislocation, and distraction: primary hemorrhage and membrane permeability in distinct mechanisms of spinal cord injury. J. Neurosurg. Spine 6, 255-266.

7. Dumont, R.J., Okonkwo, D.O., Verma, S., Hurlbert, R.J., Boulos, P.T., Ellegala, D.B., and Dumont, A.S. (2001). Acute spinal cord injury, part I: pathophysiologic mechanisms. Clin. Neuropharmacol. 24, 254-264.

8. Ergun, A., and Oder, W. (2003). Pediatric care report of spinal cord injury without radiographic abnormality (SCIWORA): case report and literature review. Spinal Cord 41, 249-253.

9. Kriss, V.M., and Kriss, T.C. (1996). SCIWORA (spinal cord injury without radiographic abnormality) in infants and children. Clin. Pediatr. (Phila) 35, 119-124.

10. Marinier, M., Rodts, M.F., and Connolly, M. (1997). Spinal cord injury without radiographic abnormality (SCIWORA). Orthop. Nurs. 16, 57-63.

11. Greaves, C.Y., Gadala, M.S., and Oxland, T.R. (2008). A threedimensional finite element model of the cervical spine with spinal cord: an investigation of three injury mechanisms. Ann. Biomed. Eng. $36,396-405$.

12. Russell, C.M., Choo, A.M., Tetzlaff, W., Chung, T.E., and Oxland, T.R. (2012). Maximum principal strain correlates with spinal cord tissue damage in contusion and dislocation injuries in the rat cervical spine. J. Neurotrauma 29, 1574-1585.

13. Thuret, S., Moon, L.D., and Gage, F.H. (2006). Therapeutic interventions after spinal cord injury. Nat. Rev. Neurosci. 7, 628-643.

14. Allen, A.R. (1911). Surgery of experimental lesion of spinal cord equivalent to crush injury of fracture dislocation of spinal column: a preliminary report. JAMA 57, 878-880.

15. Fiford, R.J., Bilston, L.E., Waite, P., and Lu, J. (2004). A vertebral dislocation model of spinal cord injury in rats. J. Neurotrauma 21, 451-458.

16. Dabney, K.W., Ehrenshteyn, M., Agresta, C.A., Twiss, J.L., Stern, G., Tice, L. and Salzman, S.K. (2004). A model of experimental spinal cord trauma based on computer-controlled intervertebral distraction: characterization of graded injury. Spine (Phila Pa 1976) 29, 23572364.

17. Maiman, D.J., Coats, J. and Myklebust, J.B. (1989). Cord/spine motion in experimental spinal cord injury. J. Spinal Disord. 2, 14-19.

18. Maiman, D.J., Myklebust, J.B., Ho, K.C., and Coats, J. (1989). Experimental spinal cord injury produced by axial tension. J. Spinal Disord. 2, 6-13.

19. Choo, A.M., Liu, J., Dvorak, M., Tetzlaff, W., and Oxland, T.R. (2008) Secondary pathology following contusion, dislocation, and distraction spinal cord injuries. Exp. Neurol. 212, 490-506.

20. Olfert, E.D., Cross, B.M., and McWilliam, A.A. (1993). Guide to the Care and Use of Experimental Animals. Canadian Council on Animal Care: Ottawa, Canada.

21. Martinez, M., Brezun, J.M., Bonnier, L., and Xerri, C. (2009). A new rating scale for open-field evaluation of behavioral recovery after cervical spinal cord injury in rats. J. Neurotrauma 26, 1043-1053.

22. Anderson, K.D., Sharp, K.G., Hofstadter, M., Irvine, K.A., Murray, M., and Steward, O. (2009). Forelimb locomotor assessment scale (FLAS): novel assessment of forelimb dysfunction after cervical spinal cord injury. Exp. Neurol. 220, 23-33.

23. Gensel, J.C., Tovar, C.A., Hamers, F.P., Deibert, R.J., Beattie, M.S., and Bresnahan, J.C. (2006). Behavioral and histological characterization of unilateral cervical spinal cord contusion injury in rats. J. Neurotrauma 23, 36-54.

24. Meyer, O.A., Tilson, H.A., Byrd, W.C., and Riley, M.T. (1979). A method for the routine assessment of fore- and hindlimb grip strength of rats and mice. Neurobehav. Toxicol. 1, 233-236. 
25. Kloth, V., Klein, A., Loettrich, D., and Nikkhah, G. (2006). Colourcoded pellets increase the sensitivity of the staircase test to differentiate skilled forelimb performances of control and 6-hydroxydopamine lesioned rats. Brain Res. Bull. 70, 68-80.

26. Metz, G.A. and Whishaw, I.Q. (2002). Cortical and subcortical lesions impair skilled walking in the ladder rung walking test: a new task to evaluate fore- and hindlimb stepping, placing, and co-ordination. J. Neurosci. Methods 115, 169-179.

27. Hamers, F.P., Lankhorst, A.J., van Laar, T.J., Veldhuis, W.B. and Gispen, W.H. (2001). Automated quantitative gait analysis during overground locomotion in the rat: its application to spinal cord contusion and transection injuries. J. Neurotrauma 18, 187-201.

28. Hobbs, C., Thoresen, M., Tucker, A., Aquilina, K., Chakkarapani, E., and Dingley, J. (2008). Xenon and hypothermia combine additively, offering long-term functional and histopathologic neuroprotection after neonatal hypoxia/ischemia. Stroke 39, 1307-1313.

29. Choo, A.M., Liu, J., Liu, Z., Dvorak, M., Tetzlaff, W., and Oxland, T.R. (2009). Modeling spinal cord contusion, dislocation, and distraction: characterization of vertebral clamps, injury severities, and node of Ranvier deformations. J. Neurosci. Methods 181, 6-17.

30. Ivancic, P.C., Pearson, A.M., Tominaga, Y., Simpson, A.K., Yue, J.J., and Panjabi, M.M. (2007). Mechanism of cervical spinal cord injury during bilateral facet dislocation. Spine (Phila Pa 1976) 32, 2467-2473.

31. Panjabi, M.M., Kifune, M., Wen, L., Arand, M., Oxland, T.R., Lin, R.M., Yoon, W.S., and Vasavada, A. (1995). Dynamic canal encroachment during thoracolumbar burst fractures. J. Spinal Disord. 8, 39-48.

32. Wilcox, R.K., Boerger, T.O., Hall, R.M., Barton, D.C., Limb, D., and Dickson, R.A. (2002). Measurement of canal occlusion during the thoracolumbar burst fracture process. J. Biomech. 35, 381-384.

33. Shahrokni, M., Zhu, Q., Liu, J., Tetzlaff, W., and Oxland, T.R. (2012). Design and biomechanical evaluation of a rodent spinal fixation device. Spinal Cord 50, 543-547.

34. Kozlowski, P., Raj, D., Liu, J., Lam, C., Yung, A.C., and Tetzlaff, W. (2008). Characterizing white matter damage in rat spinal cord with quantitative MRI and histology. J. Neurotrauma 25, 653-676.

35. McGraw, J., Gaudet, A.D., Oschipok, L.W., Steeves, J.D., Poirier, F. Tetzlaff, W., and Ramer, M.S. (2005). Altered primary afferent anatomy and reduced thermal sensitivity in mice lacking galectin-1. Pain 114, 7-18.

36. Bhatnagar, T., Liu, J., Yung, A., Cripton, P., Kozlowski, P., Tetzlaff, W., and Oxland, T. (2015). Quantifying the internal deformation of the rodent spinal cord during acute spinal cord injury-the validation of a method. Comput. Methods Biomech. Biomed. Engin. 19, 386-395.

37. Mattucci, S. (2015). Dislocation spinal cord injury in a rodent modelidentifying variability using high-speed X-ray imaging. ORS Annual Meeting, poster no. 1552.

38. King, V.M., and Apps, R. (2000). Somatotopical organization of foslike immunoreactivity in rat cervical spinal cord following noxious stimulation of the forelimb. Neuroscience 101, 179-188.

39. Watson, C., Paxinos, G., and Kayalioglu, G. (2009). The Spinal Cord: a Christopher and Dana Reeve Foundation Text and Atlas. Academic Press: London.

40. Kwon, B.K., Okon, E.B., Plunet, W., Baptiste, D., Fouad, K., Hillyer, J., Weaver, L.C., Fehlings, M.G., and Tetzlaff, W. (2011). A systematic review of directly applied biologic therapies for acute spinal cord injury. J. Neurotrauma 28, 1589-1610.

41. Tetzlaff, W., Okon, E.B., Karimi-Abdolrezaee, S., Hill, C.E., Sparling, J.S., Plemel, J.R., Plunet, W.T., Tsai, E.C., Baptiste, D., Smithson, L.J. Kawaja, M.D., Fehlings, M.G., and Kwon, B.K. (2011). A systematic review of cellular transplantation therapies for spinal cord injury. J. Neurotrauma 28, 1611-1682.

Address correspondence to: Thomas R. Oxland, PhD, PEng Department of Orthopaedics University of British Columbia 5460-818 West 10th Avenue

Vancouver, British Columbia

Canada V5Z 1 M9

E-mail: toxland@mail.ubc.ca 